\title{
Development of a One-Micrometer-Diameter Particle Size Standard Reference Material
}

\author{
G. W. Mulholland, A. W. Hartman, G. G. Hembree, \\ Egon Marx, and T. R. Lettieri \\ National Bureau of Standards, Gaithersburg, MD 20899
}

\begin{abstract}
Accepted: October 11, 1984
The average diameter of the first micrometer particle size standard (Standard Reference Material 1690), an aqueous suspension of monosized polystyrene spheres with a nominal $1 \mu \mathrm{m}$ diameter, was accurately determined by three independent techniques. In one technique the intensity of light scattered by a diluted suspension of polystyrene spheres was measured as a function of scattering angle, using a $\mathrm{He}-\mathrm{Ne}$ laser polarized in the vertical direction. The second technique consisted of measuring as a function of angle the intensity of light scattered from individual polystyrene spheres suspended in air, using a $\mathrm{He}-\mathrm{Cd}$ laser with light polarized parallel and perpendicular to the scattering plane. The measurement of row length by optical microscopy for polystyrene spheres arranged in close-packed, two-dimensional hexagonal arrays was the basis of the third technique. The measurement errors for each technique were quantitatively assessed. For the light scattering experiments, this required simulation with numerical experiments. The average diameter determined by each technique agreed within $0.5 \%$ with the most accurate value being $0.895 \pm 0.007 \mu \mathrm{m}$ based on light scattering by an aqueous suspension. Transmission electron microscopy, flow through electrical sensing zone counter measurements, and optical microscopy were also used to obtain more detailed information on the size distribution including the standard deviation $(0.0095 \mu \mathrm{m})$, fraction of off-size particles, and the fraction of agglomerated doublets $(1.5 \%)$.
\end{abstract}

Key words: array sizing; index of refraction; light scattering; Mie scattering; particle counting; particle size standards; polystyrene spheres; size distribution; transmission electron microscopy.

\section{Introduction}

This paper describes the measurements performed to accurately characterize the average particle size of the nominal one-micrometer polystyrene spheres particle size standard (Standard Reference Material 1690). The spheres were purchased from Dow Chemical Company. ${ }^{1}$ They have a nearly monosize distribution and are dispersed in water at a weight concentration of $0.5 \%$.

About the Authors: G. W. Mulholland is a research chemist in NBS' Center for Fire Research. A. W. Hartman, G. G. Hembree, Egon Marx, and T. R. Lettieri are physicists in the Bureau's Center for Manufacturing Engineering.

\footnotetext{
'Certain materials are identified in this paper in order to adequately specify the experimental procedure. Such identification does not imply recommendation or endorsement by the National Bureau of Standards, nor does it imply that the materials or equipment identified are necessarily the best available for the purpose.
}

The three techniques used for measuring average particle size were: light scattering from individual spheres suspended in air, light scattering from an aqueous suspension of the spheres, and optical row-length measurements of spheres arranged in two dimensional arrays. The selection of these techniques resulted in part from a detailed review of all the generic measurement techniques appropriate for the dimensional calibration of microscopic-particle size standards by Swyt [1] ${ }^{2}$. These same three techniques were used previously by others in characterizing a batch of monosize polystyrene spheres produced by Dow Chemical Co. (batch number LS-1028-E). Phillips et al. [2] obtained a diameter of 1.20 $\mu \mathrm{m}$ by single particle scattering measurements, Rowell et al. [3] obtained $1.21 \mu \mathrm{m}$ by measurement of light scattering from a suspension of particles, and Bierhuizen and Ferron [4] obtained $1.20 \mu \mathrm{m}$ based on array sizing. The good agreement among these independent

\footnotetext{
${ }^{2}$ Figures in brackets refer to literature references at the end of this
} paper. 
measurements gave us confidence that an accurate particle size standard was achievable.

Our study represents an extension of the earlier work in regard to the accuracy achievable by the three techniques. The major effort of our study was directed at performing the measurements in such a way as to maximize the accuracy in the determination of the mean particle size. This required the use of accurate calibration standards for magnification and angular alignment in addition to a quantitative error analysis.

Perhaps the most widely accepted technique for measuring the particle size of polystyrene spheres has been transmission electron microscopy (TEM). This is the basis of the particle size information that is provided with the monosize polystyrene spheres marketed by the manufacturer. We also had intended to use the TEM as one of our primary techniques, but we were unable to find an accurate method for calibrating the magnification of the microscope. The relatively wide range of values for particle diameter (from 1.10 to $1.26 \mu \mathrm{m}$ ) reported by five independent TEM measurements [3] for Dow Latex LS-1028-E also suggests that the method is unsatisfactory for highly accurate particle size measurements. While we did not use the TEM for determining average size, we did use it for determining the standard deviation, $\sigma_{P}$, of the size distribution.

Methods that have been used by others for measuring the particle size of polystyrene spheres include flow ultramicroscopy, ultracentrifugation, quasi-elastic scattering, and small-angle $\mathrm{x}$-ray scattering. Some general design limitations of these instruments are given below. Our choice of light scattering and optical array sizing techniques was based on their being at least competitive with other methods in regard to sizing accuracy, and on our familiarity with the measurement methods and the theoretical basis.

Both the quasi-elastic scattering technique and smallangle $\mathrm{x}$-ray scattering work best for particles smaller than about $1 \mu \mathrm{m}$. The former technique [5] measures, in effect, the particle diffusion coefficient, which increases with decreasing particle size. The resolution is limited for $1 \mu \mathrm{m}$ particles but improves with decreasing size. This is just the opposite of angular-intensity light scattering and array sizing where the resolution drops off markedly for particle sizes less than about $0.5 \mu \mathrm{m}$ diameter. Small-angle x-ray scattering [6] is also best for small particle sizes because of the small wavelengths of the $\mathrm{x}$-rays.

Davidson et al. [7] obtained an uncertainty of about $\pm 4 \%$ at the $95 \%$ confidence level for flow ultramicroscopy based on counting 300 particles. The major source of uncertainty was attributed to the statistical uncertainty in the average counting rate. The uncertainty in the number of particles counted is simply equal to the square root of that number. Van den Hul and Vanderhoff [8] obtained a mean diameter for Dow Latex LS-1028-E about $18 \%$ less than the mean of the other techniques listed by Rowell et al. [3] using ultracentrifugation. A major drawback in the technique for this application is the small density difference, 1.00 versus 1.05 , between water and the polymer. Nevertheless, Van den Hul et al. point out that all the deviation from other measurements cannot be accounted for by the uncertainty in the density difference. Apparently no detailed error analysis has been made for the ultracentrifuge applied to particle measurements.

While the major focus of our study was the accurate determination of the number average particle diameter $D_{\mathrm{n}}$, we also obtained information regarding the size distribution including the standard deviation, $\sigma_{P}$, of the particle size distribution, the fraction of off-size particles, and the fraction of agglomerated doublets. In addition, we obtained an estimate of the deviation from sphericity based on TEM measurements. A brief description of the emulsion polymerization technique for producing polystyrene spheres and the factors affecting the stability of the suspension is given in the Appendix.

\section{Light Scattering From Individual Spheres}

We determined the diameter of the polystyrene spheres by measuring the angular distribution of the intensity of the light scattered by a single sphere and comparing measured intensities with those computed for different values of the diameter, $D$, and the refractive index, $n$.

The particles were levitated electrostatically in a Differential II light-scattering photometer developed by Wyatt and Phillips [9]. This type of instrument has been used to determine $D$ and $n$ for spheres with diameters $1.099 \mu \mathrm{m}$ (Phillips et al. [2]), $0.796 \mu \mathrm{m}$ (Cook and Kerker [10]), 1.011, 0.794, and 0.600 $\mu \mathrm{m}$ (McRae [11]), and 1.01, 5.7, and $11.9 \mu \mathrm{m}$ (Davis and Ravindran [12]). The sizes given above are nominal sizes provided by Dow Chemical Company, the manufacturer of the particles. Bottiger et al. [13] have developed the capability of measuring all 16 components of the Mueller matrix, the light-scattering phase matrix, for a single polystyrene sphere. They also collected the particles on the tip of a needle for subsequent observation in an electron microscope.

A detailed error analysis enabled us to make a quantitative statement regarding the accuracy of this technique. This and other aspects of the experiment and data analysis have been previously reported by Marx and Mulholland [14]. 


\subsection{Experimental Method}

An aerosol made up of polystyrene spheres is generated by nebulizing a suspension of the spheres in distilled, filtered water; the water evaporates rapidly leaving charged spheres. One of these spheres is then levitated electrostatically in an optical cell with electrodes as shown in figure 1 . The particle is kept in the light beam of a He-Cd laser $(\lambda=441.6 \mathrm{~nm})$ by means of a servocontrol.

The scattered light reaches a photomultiplier (having an S-21 response) via a traveling periscope, which has an acceptance angle of about $2^{\circ}$ and which moves in a horizontal arc of almost $180^{\circ}$. We found that the output of the angle encoder deviates by as much as $3^{\circ}$ from the true angle, and we performed an angle calibration using an accurately indexed protractor.

We use a half-wave retardation plate to change the polarization of the incident light.

A typical sequence of measurements begins with a scan of the background, then a particle is captured and one or more scans is performed to measure the light scattered by that particle. A rotation of the half-wave plate by $45^{\circ}$ then changes the polarization of the incident beam by $90^{\circ}$, and one or more scans is performed to measure the scattered light, followed by a background measurement for the new polarization. The light intensity-angle data pair closest to each integer angle over the range $20^{\circ}$ to $160^{\circ}$ is stored on magnetic tape.

\subsection{Data Analysis}

The data are matched to the results of the calculations of the angular distribution of the intensity of the light scattered by a uniform sphere, as found by Mie and others [15-171.

The values of $D$ and $n$ are determined by minimizing the quality of fit

$$
Q(D, n)=\frac{1}{N} \sum_{i=1}^{N}\left(E_{i}-\alpha T_{i}(D, n)\right)^{2},
$$

where $N$ is the number of data points, $E_{i}$ is the measured intensity at the angle $\theta_{i}, T_{i}$ is the computed value for the same angle, and $\alpha$ is an overall scaling factor required because we do not measure absolute intensities. The expression for the $\alpha$ that minimizes $Q$ is given by

$$
\alpha=\left(\sum_{i=1}^{N} E_{i} T_{i}\right) / \sum_{i=1}^{N} T_{i}^{2}
$$

The least-squares fit is then determined by a grid search in the $D n$-plane for progressively finer grids. This procedure can be speeded up using an iterative search such as the Marquardt-Levenberg algorithm after an initial coarse grid search.

Other measures of the quality of fit are possible, and we consider mainly those that involve a change of variables. Specifically, we use the transformed intensity

$$
I_{1}(\theta)=I(\theta) \sin ^{2}(\theta / 2),
$$

because it tends to equalize the height of all the peaks for a particle of a size around $1 \mu \mathrm{m}$ in diameter. In the original variable $I(\theta)$, the large peak at small angles is overemphasized. On the other hand, the use of $I_{1}(\theta)$ overemphasizes the values at angles close to $180^{\circ}$, which are small with large relative errors.

\subsection{Results}

The theoretical best fit curves are plotted in figure 2 for a single polystyrene sphere with and without the weighting function. There are four independent determinations of the best fit diameter; two based on vertical polarization and two based on horizontal polarization data. The best fit values range from 0.898 to $0.905 \mu \mathrm{m}$, which is a range of about $0.8 \%$. A small portion of the table of values of $Q(n, D)$ near the minimum is given in
Figure 1-Single particle scattering instrument (Differential II). The pillbox shaped scattering cell has a pin electrode $\left(V_{1}\right)$ separated by an insulator from the ground plate electrode and opposite to the base plate electrode $\left(V_{2}\right)$.

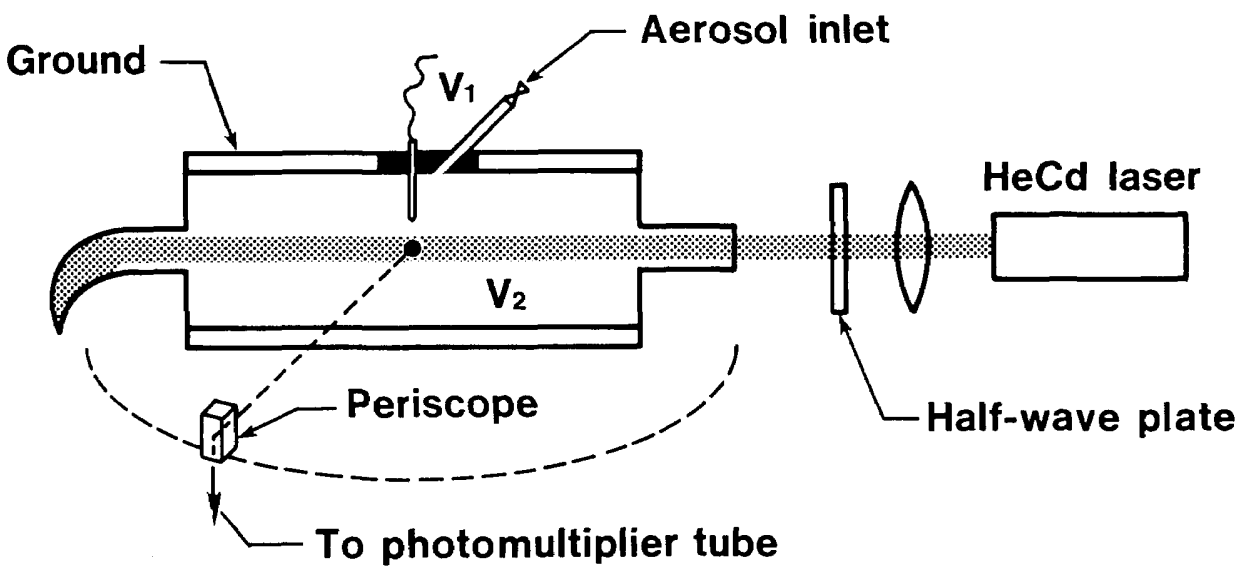



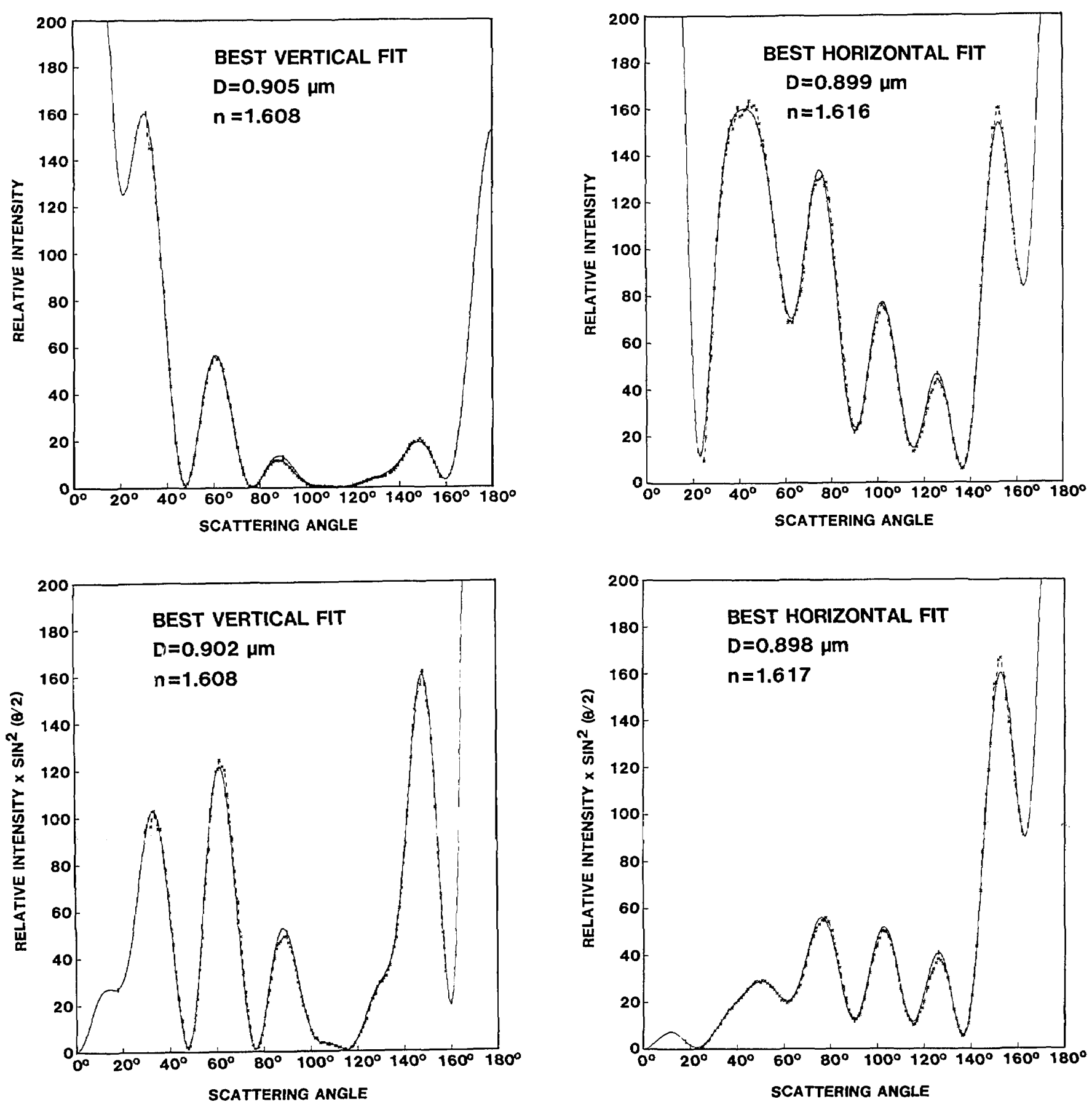

Figure 2-Best fits to the intensity of scattered light versus angle data for a single polystyrene sphere as determined by the least squares method with (bottom) and without (top) the $\sin ^{2} \theta / 2$ weighting factor. Data $(---x---x)$; theory ( - ).

table 1 . The quantity $Q$ varies rapidly with respect to $D$ for fixed $n$ (column) and rapidly with respect to $n$ for fixed $D$ (row) but is rather insensitive in the diagonal direction along which the product $n D$ is almost constant. In a three-dimensional plot of $1 / Q$ as a function of $n$ and $D$, there is a very sharp peak when the surface is viewed in one direction but almost a ridgelike appear- ance in the other as shown in figure 3. The relative insensitivity of $Q$ to correlated changes in $n$ and $D$ necessitates that careful angle calibrations and detailed data analysis be performed in order to obtain accurate values for $n$ and $D$.

The results for the eight SRM 1690 particles analyzed are contained in table 2 including the averages for the 
Table 1. Normalized values of $Q$ near the minimum for a SRM 1690 particle.

\begin{tabular}{|c|c|c|c|c|c|c|c|c|c|c|c|}
\hline \multirow[b]{2}{*}{$\begin{array}{c}n \\
D, \mu \mathrm{m}\end{array}$} & \multicolumn{4}{|c|}{ Vertical Polarization } & \multirow[b]{2}{*}{1.616} & \multirow[b]{2}{*}{$\begin{array}{c}n \\
D, \mu \mathrm{m}\end{array}$} & \multicolumn{5}{|c|}{ Horizontal Polarization } \\
\hline & 1.600 & 1.604 & 1.608 & 1.612 & & & 1.608 & 1.612 & 1.616 & 1.620 & 1.624 \\
\hline 0.899 & 4.75 & 3.18 & 2.18 & 1.70 & 1.69 & 0.893 & 14.22 & 9.78 & 6.36 & 4.07 & 3.03 \\
\hline 0.901 & 2.34 & 2.15 & 1.48 & 1.29 & 1.50 & 0.895 & 9.58 & 5.93 & 3.37 & 2.01 & 1.92 \\
\hline 0.903 & 2.31 & 1.47 & 1.09 & 1.15 & $\overline{1.58}$ & 0.897 & 5.90 & 3.11 & 1.51 & 1.16 & $\overline{2.13}$ \\
\hline 0.905 & 1.65 & 1.10 & $\underline{1}$ & $\overline{1.29}$ & 1.92 & 0.899 & 3.35 & 1.55 & $\underline{1}$ & $\overline{1.81}$ & 4.06 \\
\hline 0.907 & 1.32 & 1.06 & $\overline{1} .20$ & 1.70 & 2.52 & 0.901 & 2.15 & 1.46 & $\frac{2}{2.16}$ & 4.35 & 8.13 \\
\hline 0.909 & $1.32^{\mathrm{a}}$ & 1.33 & 1.70 & 2.40 & 3.39 & 0.903 & 2.59 & 3.24 & 5.40 & 9.25 & 14.92 \\
\hline
\end{tabular}

${ }^{\text {aT }}$ The minimum value of $Q$ in each column is underlined to illustrate the slow variation of $Q$ on a diagonal.

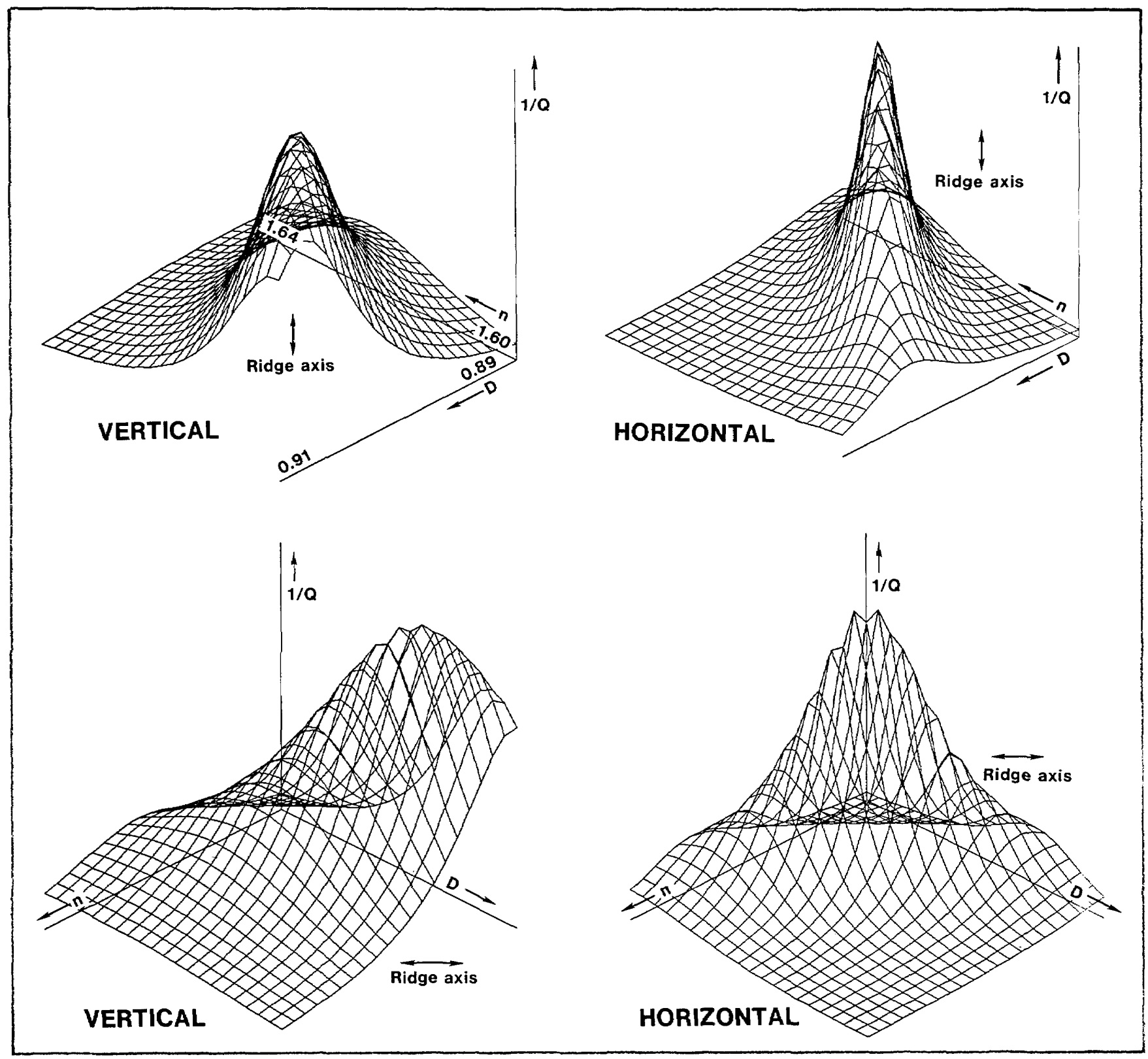

Figure 3-Two views of the three-dimensional surface of the inverse of the quality of fit, $1 / Q$, as a function of the index of refraction, $n$, and the diameter, $D$, over a range of a few percent from the best value. The ridges cause difficulties for an accurate determination of the correct values of $n$ and $D$. 
Table 2. Radius and index of refraction for eight particles from SRM 1690.

\begin{tabular}{|c|c|c|c|c|c|c|}
\hline & \multicolumn{2}{|c|}{ Harmonic Mean ${ }^{\mathrm{a}}$} & \multicolumn{2}{|c|}{$\begin{array}{l}\text { Vertical } \\
\text { Polarization }\end{array}$} & \multicolumn{2}{|c|}{$\begin{array}{c}\text { Horizontal } \\
\text { Polarization }\end{array}$} \\
\hline & $\underset{\mu \mathrm{m}}{\text { Radius }}$ & $\begin{array}{l}\text { Refractive } \\
\text { Index }\end{array}$ & $\begin{array}{l}\text { Radius } \\
\mu \mathrm{m}\end{array}$ & $\begin{array}{l}\text { Refractive } \\
\text { Index }\end{array}$ & $\begin{array}{c}\text { Radius } \\
\mu \mathrm{m}\end{array}$ & $\begin{array}{l}\text { Refractive } \\
\text { Index }\end{array}$ \\
\hline & 0.4545 & 1.614 & 0.4550 & 1.614 & 0.4535 & 1.610 \\
\hline & 0.4570 & 1.613 & 0.4570 & 1.613 & 0.4530 & 1.623 \\
\hline & 0.4490 & 1.618 & 0.4530 & 1.605 & 0.4490 & 1.618 \\
\hline & 0.4465 & 1.612 & 0.4510 & 1.600 & 0.4460 & 1.614 \\
\hline & 0.4480 & 1.613 & 0.4515 & 1.610 & 0.4480 & 1.613 \\
\hline & 0.4505 & 1.613 & 0.4535 & 1.610 & 0.4505 & 1.613 \\
\hline & 0.4535 & 1.608 & 0.4535 & 1.608 & 0.4535 & 1.610 \\
\hline & 0.4394 & 1.606 & 0.4402 & 1.603 & 0.4394 & 1.610 \\
\hline Average & 0.4498 & 1.612 & 0.4518 & 1.608 & 0.4491 & 1.614 \\
\hline$\sigma$ & 0.0051 & 0.004 & 0.0048 & 0.005 & 0.0045 & 0.004 \\
\hline
\end{tabular}

${ }^{a}$ Harmonic mean refers to the determination of the best fit radius and refractive index by taking the harmonic mean, $Q_{h}$, of the $Q$ 's for the cases of vertically and horizontally polarized light, $Q_{\mathrm{V}}$ and $Q_{\mathrm{H}}$ respectively (i.e., $2 / Q_{\mathrm{h}}=1 / Q_{\mathrm{V}}+1 / Q_{\mathrm{H}}$ ).

diameter and index of refraction, $0.900 \mu \mathrm{m}$ and 1.612 , respectively. The average particle size in this report refers to the number average defined as follows:

$$
D_{\mathrm{n}}=\frac{\sum_{i=1}^{N} D_{i}}{N},
$$

where $N$ refers to the number of particles measured. The particle size is not perfectly uniform $\left(\sigma_{P}=0.010\right)$ and we expect to see a spread in the measured diameters. On the other hand, the refractive index should be the same for all particles, and we attribute the variation to experimental error.
For one particle we took nine scans with vertically polarized light and three more with horizontal polarization. The results of the analysis are shown in table 3 . The standard deviation for the diameter, $0.0022 \mu \mathrm{m}$, is smaller than the one previously calculated for groups of particles, but that for the index of refraction, 0.005 , is about the same, suggesting that this parameter does not change, at least for particles of the same size.

\subsection{Error Analysis}

To study the effects of experimental errors on the best fit parameters, we modified the program that produces the theoretical values of the scattered intensities to add

Table 3. Results for repeated scans for one particle.

\begin{tabular}{|c|c|c|c|c|c|c|}
\hline \multirow[t]{2}{*}{ Run } & \multicolumn{2}{|c|}{ Unweighted Fit } & \multicolumn{2}{|c|}{ Weighted Fit } & \multirow[b]{2}{*}{$\begin{array}{c}\mathrm{n}=1.608^{\mathrm{a}} \\
\text { Radius } \\
\mu \mathrm{m}\end{array}$} & \multirow[b]{2}{*}{$\begin{array}{c}\mathrm{n}=1.615 \\
\text { Radius } \\
\mu \mathrm{m}\end{array}$} \\
\hline & $\begin{array}{l}\text { Radius } \\
\mu \mathrm{m}\end{array}$ & $\begin{array}{l}\text { Refractive } \\
\text { Index }\end{array}$ & $\begin{array}{l}\text { Radius } \\
\mu \mathrm{m}\end{array}$ & $\begin{array}{l}\text { Refractive } \\
\text { Index }\end{array}$ & & \\
\hline $1-V$ & 0.4402 & 1.601 & 0.4380 & 1.612 & 0.4384 & 0.4366 \\
\hline $2-V$ & 0.4364 & 1.620 & 0.4376 & 1.614 & 0.4398 & 0.4378 \\
\hline $3-V$ & 0.4392 & 1.606 & 0.4366 & 1.618 & 0.4386 & 0.4372 \\
\hline $4-V$ & 0.4402 & 1.603 & 0.4398 & 1.605 & 0.4388 & 0.4372 \\
\hline $5-V$ & 0.4394 & 1.606 & 0.4410 & 1.601 & 0.4390 & 0.4372 \\
\hline $6-V$ & 0.4380 & 1.610 & 0.4398 & 1.604 & 0.4386 & 0.4368 \\
\hline $7-V$ & 0.4398 & 1.604 & 0.4386 & 1.610 & 0.4388 & 0.4370 \\
\hline $8-V$ & 0.4394 & 1.606 & 0.4388 & 1.609 & 0.4390 & 0.4372 \\
\hline $9-V$ & 0.4394 & 1.607 & 0.4400 & 1.604 & 0.4392 & 0.4374 \\
\hline $1-\mathrm{H}$ & 0.4394 & 1.610 & 0.4402 & 1.601 & 0.4398 & 0.4384 \\
\hline $2-\mathrm{H}$ & 0.4380 & 1.612 & 0.4402 & 1.612 & 0.4386 & 0.4374 \\
\hline $3-\mathrm{H}$ & 0.4384 & 1.614 & 0.4364 & 1.620 & 0.4394 & 0.4382 \\
\hline Average & 0.4390 & 1.608 & 0.4389 & 1.609 & 0.4390 & 0.4374 \\
\hline$\sigma$ & 0.00110 & 0.0053 & 0.00150 & 0.0063 & 0.00047 & 0.00053 \\
\hline
\end{tabular}

${ }^{2}$ The last two columns correspond to values of the radii obtained for a fixed value of the refractive index (unweighted fit). The first value is obtained for the best fit to all particles of this size, and the second one is the published bulk value. 
simulated errors and generate a file of "data" to be processed by the same procedure as described above. Noise obtained using a random number generator with a maximum amplitude of $1-3 \%$ of the intensity at each angle was added to all curves.

The major component of the random error is attributed to the noise in the intensity of the scattered light. This noise can be monitored by fixing the angle and recording a trace of the intensity versus time. The general characteristics of such a trace show that there are two components of this noise. The smaller component, about $2 \%$ of the intensity, affects each angle reading independently; the larger component, which we think comes from the motion of the particle within the laser beam when the servocontrol is activated, is about $5 \%$ and has a lower frequency so that it affects intensity readings in groups of about 10 degrees for the data collection speed that we used. The uncertainty in particle size for a $0.900 \mu \mathrm{m}$ particle diameter was found to be at most $0.3 \%$ or $0.0027 \mu \mathrm{m}$. As described above, the observed variability in particle diameter for 12 repeat scans of the same particle resulted in a $\sigma$ of $0.0022 \mu \mathrm{m}$, which is consistent with most of the error being attributed to noise in the intensity of the scattered light.

The quantity of primary interest is the random error associated with the determination of the average particle diameter. The standard deviation of the size distribution, $\sigma_{P}$, is much larger than the $\sigma$ associated with repeat measurements and will ultimately limit the accuracy in the mean size determination. Our measured $\sigma_{P}$ of the particle diameters based on eight particles is 0.010 $\mu \mathrm{m}$; an estimate based on our electron microscopy for about 160 particles, as discussed below, is slightly smaller, $0.0095 \mu \mathrm{m}$. The component of the overall uncertainty in particle diameter resulting from the width of the size distribution is given by

$$
R=t_{m}(0.025) \frac{\sigma_{P}}{\sqrt{n}},
$$

where $n$ is the number of particles (eight in this case) and $t_{m}(0.025)$ is the Student $t$-value for $m$ degrees of freedom and for $95 \%$ confidence level. The number of degrees of freedom relates to the determination of $\sigma_{P}$ and is one less than the number of particles sized by electron microscopy, 159. We obtain a value of $R$ equal to $0.0066 \mu \mathrm{m}$.

The sources of systematic errors include angle drift, $\delta_{\theta}$, polarization misalignment, $\delta_{\mathrm{P}}$, wavelength uncertainty, $\delta_{\lambda}$, and non-volatile impurities, $\delta_{\mathrm{I}}$. There is an error on the order of $2 \%$ if the angle encoder output is not calibrated. To this is added the problem of a slight drift in the calibration that was apparently electronic in origin. Over the two days during which the light scattering measurements were performed on the eight polystyrene spheres, the angle drift was about $0.08^{\circ}$. Includ- ing this drift in a numerical simulation, we find the uncertainty in particle size due to the drift, $\delta_{\theta}$, to be $0.0028 \mu \mathrm{m}$.

The mixture of polarization states comes primarily from imperfections in the optical components and the misalignment of the periscope. The imperfections in the half-wave plate and other optical components allow the leakage of a small fraction of light (1 part in 200) at the orthogonal polarization direction. The error from the inclination of the periscope with respect to the scattering plane is comparable to the error due to the optical imperfections. A combined estimate of these effects comes to $\delta_{\mathrm{P}}=0.0018 \mu \mathrm{m}$.

The least important error is the uncertainty in the wavelength of the He-Cd laser, $\delta_{\lambda}=0.00007 \mu \mathrm{m}$.

In addition to instrumental errors, there are uncertainties associated with the nature of the particle. While the polystyrene spheres are often considered to be homogeneous, we expect the existence of a surface coating from the non-volatile emulsifier added during the preparation of the particles. If we assume that all the emulsifier resides on the particle surface, we find that the diameter is increased by $0.0008 \mu \mathrm{m}$, so that $\delta_{1}=-0.0008$. There is also a contribution to the surface coating from the 2 ppm impurities in the distilled water in which the particles are suspended, but it is negligible in comparison with the emulsifier contribution.

An estimate of the total uncertainty, $U_{\mathrm{T}}$, is obtained by adding the random error, $R$, to the sum of the absolute values of the systematic errors.

$$
U_{\mathrm{T}}=R+\left|\delta_{\theta}\right|+\left|\delta_{\mathrm{P}}\right|+\left|\delta_{\mathrm{I}}\right|
$$

Using the values given above, we obtain $U_{\mathrm{T}}=0.012 \mu \mathrm{m}$ with a number average size of $0.900 \mu \mathrm{m}$.

The above analysis includes effects for which we have quantitative estimates. Two other possible systematic error sources are slight asphericity of the particle and possible inhomogeneity of the particle resulting from strain as the water evaporates from the particle. Transmission electron microscope measurements of the particle described in section 5 indicate an asphericity of $0.6 \%$ at a precision of about $0.3 \%$. Over the course of our measurements the particle would have undergone extensive rotation as a result of Brownian motion. We have no quantitative estimate of this effect, though we intuitively expect our value to be close to the volume equivalent sphere diameter.

\section{Light Scattering From a Suspension of Spheres}

Light scattering measurements on a suspension of particles is complementary to single-particle scattering measurements. The signal-to-noise performance for sin- 
gle-particle measurements is limited by the small scattering intensity and by particle movement in the beam. For scattering from a particle suspension the signal-to-noise ratio is much improved because of the large number of particles in the scattering volume and because of the use of photon counting. An accurately indexed rotary table was used for our light-scattering measurements with a suspension, whereas a rapidly-traversing periscope with some drift in the angle calibration was used for the single-particle measurements. One disadvantage of measurements with a suspension is that there is one more unknown in the measurement, namely the size distribution, compared to the single-particle measurements where particle size and the index of refraction are the only unknowns.

There have been a number of studies of light scattering by nearly monosized suspensions. Kerker's book [17] includes several studies of the determination of the mean size and standard deviation based on the angular dependence of the polarization ratio. Our instrument design is similar to the one described by Wims and Myers [18]. The recent determination of particle size of polystyrene spheres by Rowell et al. [3] by light scattering measurements is similar to our study in terms of measurement technique and data analysis.

\subsection{Design of the Photometer}

A schematic diagram of the light scattering photometer for particle suspension is shown in figure 4. A $5-\mathrm{mW}$ intensity-stabilized He-Ne laser beam is directed to the center of a $10-\mathrm{cm}$ diameter scattering cell with optical flat windows on opposite sides. Incident light is vertically polarized and only the vertically polarized component of the scattered light is detected (VV scattering). A beam stop is positioned in the cell to minimize the amount of reflected light reaching the detector. The collection optics (figs. 4 and 5) consist of two apertures, a $1 \times$ microscope objective, a vertical polarizer, and a glass diffusing screen. The scattered light is detected by a low-noise photomultiplier tube with a small $(2.5 \mathrm{~mm}$ diameter) photo cathode. The output pulses from the anode are amplified, discriminated, and then counted over a 10 -second time interval. The typical number of counts per 10-second interval at the first peak is about 200,000 , with repeat measurements agreeing within about $1 \%$. The lowest count was on the order of 10,000 .

For some measurements, part of the laser beam is split off and sent into a reference photomultiplier. Output counts from the reference amplifier-discriminator are used as clock pulses to the photon counter. In this manner, a ratio is taken between the signal photon counts and reference photon counts, thus cancelling out the laser intensity fluctuations. This is not a significant effect for the intensity stabilized He-Ne laser, but it is significant for the He-Cd laser to be discussed later.

A rotary table with an accuracy and reproducibility of \pm 1 arc second $\left(0.0003^{\circ}\right)$ is used for the angle measurements. The rotary table is indexed at two-degree increments. The PMT is mounted rigidly to an arm attached to the rotary table. Using a micrometer adjustment and a dial indicator, the center of the scattering cell is made coincident with the center of rotation of the
Figure 4-Light scattering photometer for particle suspension. Optical windows not shown.

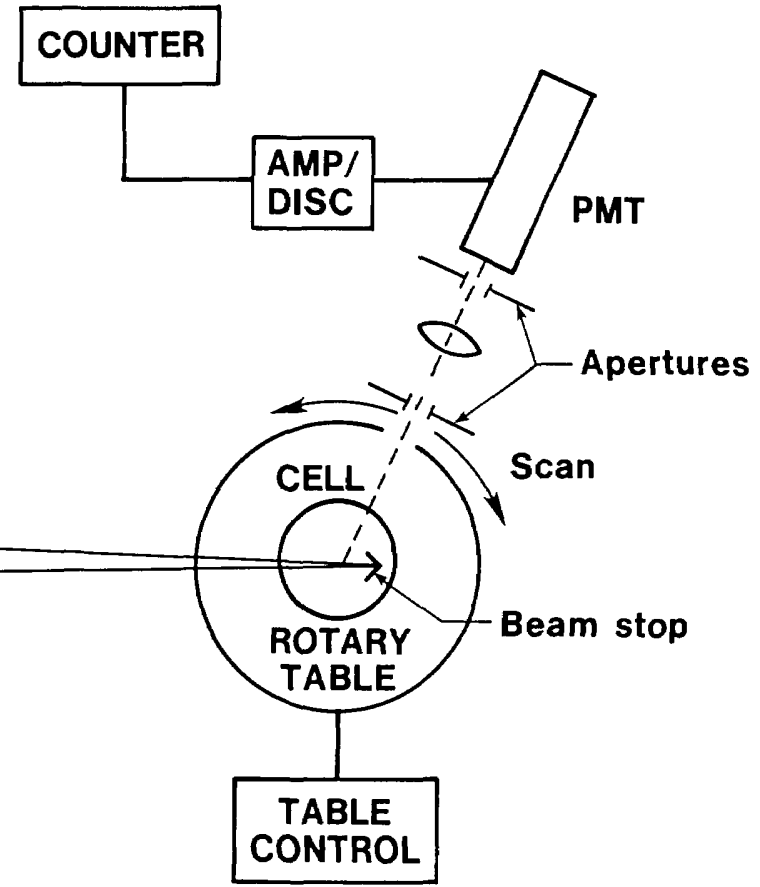




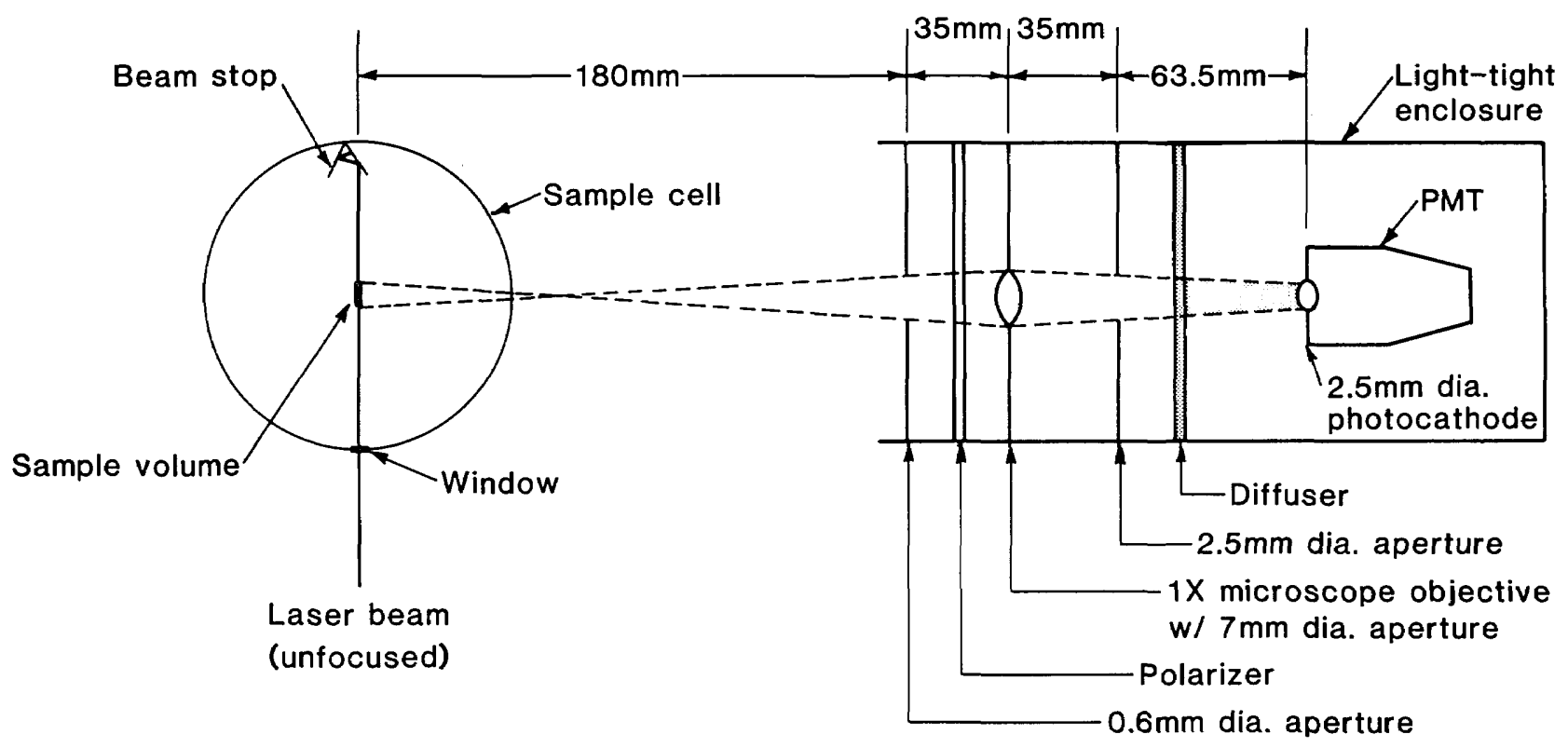

Figure 5-Design of detector optics for minimizing acceptance angle.

rotary table. Next the laser is mounted so that the beam goes through the center of the two optical flats and reflects back on itself. The pinholes in the detector system are then positioned in the center of the laser beam. The estimated deviation of the zero angle alignment, including a slight error in positioning the cell (about 0.05 $\mathrm{mm}$ ) and in the pinhole positioning (about $0.2 \mathrm{~mm}$ ), leads to an uncertainty in the zero angle of about $0.07^{\circ}$. The effect of this uncertainty on the particle size determination will be discussed in subsection 3.4.

It is important to have a small acceptance angle for the detector in order to obtain good size resolution. This is accomplished in our design by using two pinholes before the detector as indicated in figure 5 . In addition, a collection lens is used to limit the depth of field. With these optics in the collection arm, the measured acceptance angle is about $\pm 1^{\circ}$ as measured at the $70 \%$ transmission points. This compares favorably with the calculated acceptance angle of $\pm 1.3^{\circ}$ at the zero transmission points. The two-degree acceptance angle gives a calculated sample volume on the order of $1.3 \times 10^{-2} \mathrm{~cm}^{3}\left(13 \mathrm{~mm}^{3}\right)$.

\subsection{Sample Preparation and Experimental Procedure}

Clean glassware and "particle free" water are used to minimize extraneous particles in the suspension. After each experiment, the cell is washed with detergent, rinsed, and then cleaned in hot nitric acid for a couple of hours. After rinsing the cell and cover glass 8 to 10 times with deionized, filtered water, the cell is dried without water marks by directing a particle-free flow of air over the external surface of the cell. Deionized water with a resistivity of about 18 megohm-centimeter is used for rinsing glassware and for sample dilution. A pleated membrane filter with a $0.2-\mu \mathrm{m}$ pore size is also used on the water outlet to remove particulates.

In order to minimize multiple scattering, the particle suspension is diluted to a concentration on the order of $10^{6}$ particles $/ \mathrm{cm}^{3}$. Dilution of approximately four orders of magnitude is required to dilute the standard samples, which are $0.5 \%$ by weight.

After positioning the optical cell, a beam stop is inserted in the cell to minimize reflected light. Measurements are performed every two degrees from $20^{\circ}$ to about $140^{\circ}$, requiring about 30 minutes per experiment. At each angle, two intensity measurements are made to check for consistency and to avoid spurious data resulting from contamination particles which occasionally enter the scattering volume.

\subsection{Data Analysis}

For the case of light scattering from a suspension, there are three unknown parameters: the numberaverage size, $D_{\mathrm{n}}$, the standard deviation of the size distribution, $\sigma_{P}$, and the index of refraction of the polystyrene sphere. We assume the size distribution to be normal. For the narrow size distribution of interest here, there is little difference between a log-normal distribution, which others have assumed for the polystyrene spheres, and a normal distribution. Determinations of size distributions of polystyrene spheres by electron microscopy support this assumption. In carrying out our data analysis, we assume the index of refraction to be known, and treat $D_{\mathrm{n}}$ and $\sigma_{P}$ as unknown parameters. In 
subsection 3.5 we consider the sensitivity of the results to the assumed value of the index of refraction.

For a distribution of particle sizes, the theoretically predicted scattering intensity, $I\left(\theta_{i}, D_{\mathrm{n}}, n\right)$, is the singleparticle scattering intensity, $T\left(\theta_{i}, D_{\mathrm{j}}, n\right)$, averaged over the size distribution and the acceptance angle of the detector.

$$
\begin{gathered}
I\left(\theta_{i}, D_{\mathrm{n}}, \sigma_{P}\right)=\frac{1}{\sqrt{(2 \pi) \sigma_{P}}} \sum_{j=1}^{m} \exp \left[-\frac{\left(D_{\mathrm{j}}-D_{\mathrm{n}}\right)^{2}}{2 \sigma_{P}^{2}}\right]\left\{T\left(\theta_{i}, D_{\mathrm{j}}, n\right)\right. \\
\left.+0.7 T\left(\theta_{i}-1, D_{\mathrm{j}}, n\right)+0.7 T\left(\theta_{i}+1, D_{\mathrm{j}}, n\right)\right\}
\end{gathered}
$$

where $m$ represents the number of size increments and the terms with $\theta_{i}+1$ and $\theta_{i}-1$ take into account the effects due to the finite acceptance angle of the detector. The quality of fit, $Q$, for the particle suspension experiment is defined by the same type of expression as eq (1),

$$
Q=\frac{1}{N} \sum_{i=1}^{N}\left[E_{i}-\alpha I\left(\theta_{i}, D_{\mathrm{n}}, \sigma_{P}\right)\right]^{2},
$$

where the index $i$ refers to angle, and $E_{i}$ is the measured scattered intensity at $\theta_{i}$. The expression for $\alpha$ is the same as given by eq (2) but with $I\left(\theta_{i}\right)$ replacing $T_{i}$.

For the refractive index of the polystyrene spheres, we use the value 1.588 for bulk polystyrene at $\lambda=632.8$ $\mathrm{nm}$ given by Boundy and Boyer [19]. The refractive index of water at $23^{\circ} \mathrm{C}$ was taken to be 1.3315 based on measurements by Tilton and Taylor [20]. The appropriate wavelength and index of refraction for a particle suspended in water are the ratios of the respective values in vacuum to the refractive index of water giving

$$
\begin{aligned}
& \frac{\lambda_{\text {vac }}}{n_{\text {water }}}=0.4752 \mu \mathrm{m} \\
& \frac{\mathrm{n}_{\mathrm{PSL}}(\mathrm{vac})}{\mathrm{n}_{\text {water }}}=1.1925 .
\end{aligned}
$$

The number of intervals in the size distribution, $m$, is typically taken to be 10 or 20 . The size increment, $\Delta$, from $D_{\mathrm{j}}$ to $D_{\mathrm{j}+1}$ is chosen to be

$$
\Delta=\frac{4 \sigma_{P}}{m-1}
$$

so that the average in eq ( 7 ) covers the full size distribution regardless of the value of $\sigma_{p}$. For a specified value of $D_{\mathrm{n}}$ and $\sigma_{P}$, the quantity $I$ is calculated at two degree intervals from $20^{\circ}$ to $140^{\circ}$. The value of $Q$ is determined from eqs (8) and (2). The best-fit values for $D_{\mathrm{n}}$ and $\sigma_{p}$ are determined by finding the minimum $Q$ for a range of values for $D_{\mathrm{n}}$ and $\sigma_{P}$. The range for $D_{\mathrm{n}}$ is typically $0.893-0.898 \mu \mathrm{m}$ in increments of $0.0005 \mu \mathrm{m}$, and, for $\sigma_{P}$, from 0.021 to $0.039 \mu \mathrm{m}$ in increments of $0.002 \mu \mathrm{m}$. Typ- ical experimental data and best-fit results are shown in figure 6.

We also consider a second definition of quality of fit based on weighting the intensities by a function of the angle. Specifically, we consider

$$
I_{1}\left(\theta_{i}\right)=I\left(\theta_{i}\right) \sin ^{4}\left(\frac{\theta_{i}}{2}\right)
$$

which reduces the contributions to the quality of fit from the large peaks at small scattering angles. As seen in figure 7, which is based on the same data as figure 6 , the weighted peaks and valleys are approximately uniform in amplitude throughout the angular range. The

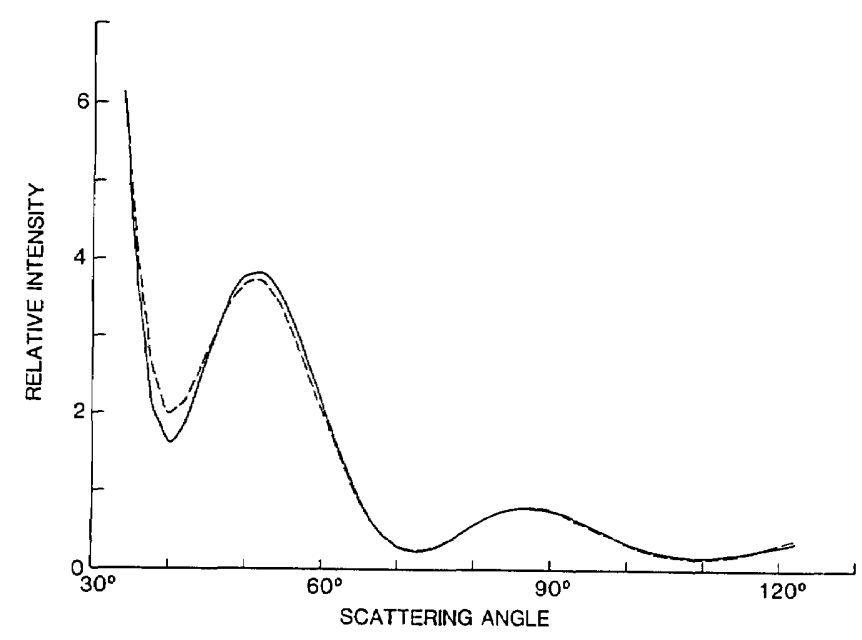

Figure 6-Best fit curve for the intensity of scattered light as a function of angle with $D_{\mathrm{n}}=0.893 \mu \mathrm{m}$ and $\sigma_{p}=0.027 \mu \mathrm{m}$. Data $(---)$; theory (—).

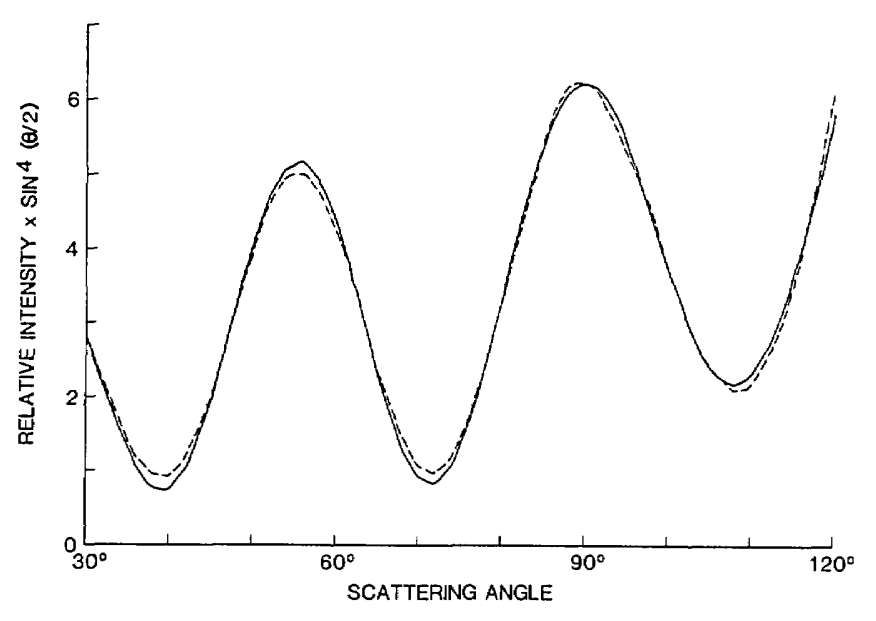

Figure 7-Best fit curve for the intensity of scattered light weighted by $\sin ^{4}(\theta / 2)$ as a function of angle with $D_{\mathrm{n}}=0.896 \mu \mathrm{m}$ and $\sigma_{p}=0.023$ $\mu \mathrm{m}$. The experimental data is the same as for figure 6. Data $(---)$; theory (—). 
values of $Q$ as a function of $D_{\mathrm{n}}$ and $\sigma_{P}$ are given in table 4 for this case (experiment A3). We consider this second definition of quality of fit to be superior to the first because all the data are significant. For our data, both techniques produce very nearly the same result; for example, $D_{\mathrm{n}}$ for the first method is 0.894 compared to 0.896 for the second method.

\subsection{Results}

One drop from one of the SRM 1690 vials, selected at random from 1000 vials, was used in preparing each of the 10 samples. Light-intensity-versus-angle data were taken for each sample and the best-fit parameters were calculated. The results of the measurements are presented in table 5 . The average value of $D_{\mathrm{n}}, \bar{D}_{\mathrm{n}}$, for experiments A2 through $\mathrm{J} 1$ was found to be $0.895 \mu \mathrm{m}$ with a standard deviation associated with the average size, $\sigma_{D_{\mathrm{n}}}$, of 0.0007 . The average value of the standard deviation of the size distribution, $\sigma_{P}$, was found to be $0.029 \mu \mathrm{m}$. Also indicated in table 5 are four repeat mea-

Table 4. The quality of fit, $\mathrm{Q}$, for experiment $\mathrm{A} 3$ with weighting factor

\begin{tabular}{|c|c|c|c|c|c|}
\hline & $\sigma_{P}, \mu \mathrm{m}$ & 0.021 & 0.023 & 0.025 & 0.027 \\
\hline \multicolumn{6}{|l|}{$\mathrm{D}, \mu \mathrm{m}$} \\
\hline 0.8945 & & 0.158 & 0.147 & 0.149 & 0.165 \\
\hline 0.8950 & & 0.147 & 0.140 & 0.146 & 0.167 \\
\hline 0.8955 & & 0.140 & 0.137 & 0.148 & 0.172 \\
\hline 0.8960 & & 0.139 & 0.140 & 0.154 & 0.181 \\
\hline 0.8965 & & 0.143 & 0.147 & 0.164 & 0.192 \\
\hline
\end{tabular}

Table 5. Best-fit values for $D_{\mathrm{n}}$ for 10 samples of SRM 1690 based on light scattering from a particle suspension.

\begin{tabular}{ccccc}
\hline \hline & & \multicolumn{3}{c}{$\begin{array}{c}\text { photon } \\
\text { counts } / 10 \mathrm{~s}^{\mathrm{d}}\end{array}$} \\
Sample $^{\mathrm{a}}$ & $D_{\mathrm{n}, \mu \mathrm{m}}$ & $\sigma$ & $Q$ \\
\hline A2 & 0.8945 & 0.029 & 197,000 & 0.276 \\
B & 0.8965 & 0.025 & 165,000 & 0.287 \\
C & 0.8945 & 0.031 & 177,000 & 0.321 \\
D & 0.8940 & 0.031 & 179,500 & 0.272 \\
E & 0.8955 & 0.029 & 172,900 & 0.288 \\
& & & & \\
F & 0.8950 & 0.029 & 150,500 & 0.224 \\
G & 0.8945 & 0.027 & 166,000 & 0.246 \\
H & 0.8950 & 0.027 & 97,500 & 0.166 \\
I & 0.8945 & 0.031 & 142,700 & 0.231 \\
J1 ${ }^{\text {b }}$ & 0.8955 & 0.029 & 137,200 & 0.280 \\
& & & & \\
J2 & 0.8950 & 0.029 & 137,000 & 0.221 \\
J3 & 0.8955 & 0.027 & 137,500 & 0.234 \\
J4 & 0.8955 & 0.029 & 136,000 & 0.217 \\
A3 & 0.8955 & 0.023 & 167,500 & 0.137 \\
\hline
\end{tabular}

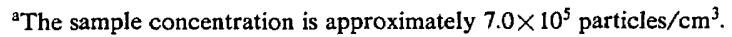

${ }^{b}$ The numerals refer to repeat measurements of the same sample.

'Sample A3 was prepared and the measurements made six months after the other measurements.

${ }^{d}$ Measured at $30^{\circ} \mathrm{C}$. surements for one sample with the scans performed sequentially over a period of about three hours. The standard deviation for the average size for the repeat measurements is less than $0.0003 \mu \mathrm{m}$. We think that the larger standard deviation obtained for the 10 samples is a result of the sample preparation and cell positioning and not of differences in the vials; in any event, both standard deviations are small and represent a minor component of the overall error as discussed in subsection 3.5.

Scattering measurements were also performed with a He-Cd laser with $\lambda=441.6 \mathrm{~nm}$, as a test for consistency. The appropriate values for the indices of refraction of water and polystyrene for this wavelength at $23{ }^{\circ} \mathrm{C}$ are 1.3395 and 1.615, respectively. Because of the lower intensity stability of the He-Cd laser, the ratio of signal photon counts to reference counts was taken as described in subsection 3.1. In this case $D_{\mathrm{n}}$ was found to be 0.896 with $\sigma_{P}=0.031$.

\subsection{Error Analysis}

The major elements of the random error component of the overall uncertainty are the photon counting noise and the fluctuation in the number of particles in the scattering volume. The percentage noise arising from the statistical nature of photon counting goes as $1 / \sqrt{N}$ so that even for a low count of 10,000 the noise is only $1 \%$. Another $1 / \sqrt{N}$ term is caused by the fluctuation in the number of particles in the scattering volume. For a number concentration of $7 \times 10^{5}$ particles $/ \mathrm{cm}^{3}$ and a typical scattering volume of $1.3 \times 10^{-2} \mathrm{~cm}^{3}$, the number of particles is $9.1 \times 10^{3}$ leading to a noise of $1.0 \%$. Assuming the two fluctuations to be independent, we obtain a combined noise of $1.5 \%$. The effect of this noise was simulated by the same technique as described in subsection 2.4 and found to change the particle size by $0.0006 \mu \mathrm{m}$. This is comparable to the observed $\sigma$, 0.0007 , for the measurements with the 10 samples. The random component of the uncertainty is calculated as in subsection 2.4,

$$
R=t_{n-1}(0.025) \frac{\sigma}{\sqrt{n}}
$$

where $n$ refers to the number of samples. In this case the number of degrees of freedom is $n-1$. We obtain for $R$ a value of $0.0005 \mu \mathrm{m}$, which is a factor of 13 less than for the single-particle measurements.

The primary component of the systematic error is uncertainty in the refractive index of the particle with lesser contributions from multiple scattering, finite acceptance angle of detector, misalignment of detector, and reflected light. The uncertainty in the angle mea- 
surement and in the wavelength of light is negligible in comparison to these others.

The values of the refractive index of polystyrene spheres obtained by various investigators were extrapolated to the $\mathrm{He}-\mathrm{Ne}$ laser wavelength using the following dispersion equation:

$$
n=A+\frac{B}{\lambda^{2}}
$$

The constants $A$ and $B$ are obtained from the measured value of $n$ at one wavelength together with Starkie's [21] value of 31.0 for the dispersion ratio defined as

$$
\frac{\mathrm{n}_{5893}-1}{\mathrm{n}_{4861}-\mathrm{n}_{6563}}=31 \text {. }
$$

The resulting values given in table 6 are close to the bulk value of 1.588 but, based on these data, we cannot rule out the possibility of the refractive index of the particle being slightly different from that of the bulk. The range of values obtained for liquid suspensions of the particles

\begin{tabular}{|c|c|c|c|}
\hline Investigator & $n$ & $\lambda, \mu \mathrm{m}$ & $\mathrm{n}(0.6328)^{\mathrm{a}}$ \\
\hline \multicolumn{4}{|l|}{ Boundy and Boyer } \\
\hline bulk value & & & 1.588 \\
\hline \multicolumn{4}{|l|}{ Heller and Pugh } \\
\hline $0.132-0.295 \mu \mathrm{m}$ & $1.602 \pm 0.008$ & 0.5461 & 1.594 \\
\hline \multicolumn{4}{|l|}{ Smart and Willis } \\
\hline $0.132-0.295 \mu \mathrm{m}$ & 1.581 & 0.589 & 1.577 \\
\hline \multicolumn{4}{|l|}{ Marx and Mulholland } \\
\hline $\mathrm{D}=0.460 \mu \mathrm{m}^{\mathrm{b}}$ & $1.603 \pm 0.029$ & 0.4416 & 1.579 \\
\hline $\mathrm{D}=0.600 \mu \mathrm{m}$ & $1.599 \pm 0.013$ & 0.4416 & 1.575 \\
\hline $\mathrm{D}=0.914 \mu \mathrm{m}$ & $1.612 \pm 0.004$ & 0.4416 & 1.588 \\
\hline $\mathrm{D}=1.101 \mu \mathrm{m}$ & $1.619 \pm 0.002$ & 0.4416 & 1.595 \\
\hline \multicolumn{4}{|l|}{ McRae } \\
\hline $\mathrm{D}=0.600 \mu \mathrm{m}^{\mathrm{b}}$ & $1.63 \pm 0.019$ & 0.5145 & 1.618 \\
\hline $\mathrm{D}=0.794 \mu \mathrm{m}$ & $1.58 \pm 0.008$ & 0.5145 & 1.569 \\
\hline $\mathrm{D}=1.011 \mu \mathrm{m}$ & $1.57 \pm 0.012$ & 0.5145 & 1.559 \\
\hline \multicolumn{4}{|l|}{ Davis and Ravindran } \\
\hline $\mathrm{D}=11.9$ and $5.7 \mu \mathrm{m}$ & & & 1.575 \\
\hline \multicolumn{4}{|l|}{$\begin{array}{l}\text { Bottiger, Voss, } \\
\text { and Fry }\end{array}$} \\
\hline $\mathrm{D}=1.112 \mu \mathrm{m}$ & 1.615 & 0.4416 & 1.590 \\
\hline \multicolumn{4}{|l|}{$\begin{array}{l}\text { Phillips, Wyatt, } \\
\text { and Berkman }\end{array}$} \\
\hline $\mathrm{D}=1.099 \mu \mathrm{m}^{\mathrm{b}}$ & $1.59 \pm 0.001$ & 0.5145 & 1.578 \\
\hline
\end{tabular}

Table 6. Refractive index of polystyrene.

${ }^{\mathrm{a}}$ The refractive index has been extrapolated to $\lambda=0.6328$ using the equation $n=A+B / \lambda^{2}$.

${ }^{\text {'}}$ The values of $D$ are the values obtained by Dow Chemical Co. using transmission electron microscopy. extends from 1.577 to 1.594 and is based, respectively, on transmitted light intensity measurements by Smart and Willis [22] and interferometric measurements by Heller and Pugh [23]. In both cases the data analysis is the most straightforward for small particle sizes, and only the small particle size results are given in table 6 .

All the other values in the table correspond to lightscattering measurements of single particles levitated in air. We consider Marx and Mulholland's value [14] of 1.588 for the nominal $0.914 \mu \mathrm{m}$ particles to be the best of the single-particle measurements in regard to SRM 1690 , since the measurements were made on the SRM particles and great care was taken with regard to angle calibration and data analysis. The low value obtained by McRae [11] might have resulted from an error in the calibration of the angle encoder. We observed an apparent size-dependence of the refractive index similar to that reported by McRae when our angle calibration was off by about $3^{\circ}$. The measurements of Davis and Ravindran [12] were done on much larger polystyrene spheres $(5.7$ and $11.9 \mu \mathrm{m})$ and the quality of the data appears to be lower than that of the other studies. The poorer data may have resulted from a slight asphericity or inhomogeneity in the particles. We take as our range of uncertainty in refractive index 1.577 to 1.595 , which encompasses all the values in table 6 except the values of Davis and Ravindran, McRae, and Marx and Mulholland's value for the $0.600-\mu \mathrm{m}$ particle size which had a large uncertainty.

To estimate the effect of this uncertainty on $D_{n}$, we repeated the data analysis for experiment $\mathrm{A} 3$ using the two extreme values of $n$. The value 1.577 leads to a slightly increased value of $D_{n}, 0.899$, while the larger value of 1.595 leads to a slightly reduced mean diameter of $0.893 \mu \mathrm{m}$. So the contribution to the uncertainty from this effect, $\delta_{n}$, equals \pm 0.003 .

The effect of multiple scattering on the results was estimated by performing measurements at two concentrations. According to the criterion established by Napper and Ottewill [24], multiple-scattering effects become insignificant for the ratios of the average distance between particles, $l$, and the particle diameter, $D$, greater than 100. For concentrations of $7.0 \times 10^{5}$ and $3.5 \times 10^{6}$ particles $/ \mathrm{cm}^{3}$, we find that $l / D$ is 125 and 70 respectively. Frorh analyzing the light-scattering data for these two cases, we obtain $0.894 \mu \mathrm{m}$ at the lower concentration and $0.890 \mu \mathrm{m}$ at the higher concentration. We estimate $\delta_{\mathrm{m}}$ as $+0.001 \mu \mathrm{m}$ allowing for a slight residual effect even though the criterion given above is satisfied for the 10 samples analyzed.

The effect of the finite acceptance angle of the photometer was determined by computer simulation in which a slit width integration was performed over the theoretically-predicted intensity pattern for $D_{\mathrm{n}}=0.900$ 
and $\sigma_{P}=0.010 \mu \mathrm{m}$. The "data" were then analyzed by the same procedure with the intensity weighted by $\sin ^{4}(\theta / 2)$, and the resulting values of $D_{\mathrm{n}}$ and $\sigma_{P}$ were 0.900 and $0.011 \mu \mathrm{m}$ respectively. So we find $\delta_{\theta}$ to be at most $0.0005 \mu \mathrm{m}$. If the intensities were analyzed directly without the angular weighting function, the values of $D_{\mathrm{n}}$ and $\sigma_{P}, 0.899$ and 0.015 , changed more as a result of the finite acceptance angle than the values obtained using the intensity weighted by the angular function. We also used computer simulation to estimate the error associated with a slight misalignment at zero angle, about $0.07^{\circ}$, and found $\delta_{\mathrm{D}}$ to be $0.0004 \mu \mathrm{m}$.

Without a beam stop, on the order of $4 \%$ of the laser beam is reflected from the glass-air interface back into the cell. This would lead to some fraction of the light reaching the detector having been scattered through the supplement of the scattering angle. We obtain an increase of $0.002 \mu \mathrm{m}$ size without the beam stop and estimate an error, $\delta_{k}$, of at most $+0.001 \mu \mathrm{m}$ due to residual reflection.

As discussed in section 5, about $1.5 \%$ of the particles is in the form of agglomerated doublets. Bottiger et al. [13], having measured all 16 components of the Mueller matrix for a doublet of $1.112 \mu \mathrm{m}$ polystyrene spheres, find that the peaks and valleys in the scattering pattern appear at the same angles as for the single sphere but that the amplitudes are smaller. We have no quantitative estimate of this agglomeration error, $\delta_{\mathrm{A}}$, but we intuitively expect it to be less than $0.001 \mu \mathrm{m}$.

Estimating the total uncertainty in the same manner as in subsection 2.4, we obtain an expression for $U_{\mathrm{T}}$ shown below together with estimates of the different terms,

$$
\begin{gathered}
U_{\mathrm{T}}=R+\left|\delta_{\mathrm{n}}\right|+\left|\delta_{\mathrm{m}}\right|+\left|\delta_{\theta}\right|+\left|\delta_{\mathrm{D}}\right|+\left|\theta_{\mathrm{R}}\right|+\left|\delta_{\mathrm{A}}\right| \\
0.00050 .0030 .0010 .00050 .00040 .0010 .001
\end{gathered}
$$

Using these estimates, we obtain $U_{\mathrm{T}}=0.007 \mu \mathrm{m}$ with a number-average size, $D_{\mathrm{n}}$, of $0.895 \mu \mathrm{m}$. The uncertainty is lower than for the single-particle measurement because of the much smaller random component of error, $0.0005 \mu \mathrm{m}$ compared to $0.007 \mu \mathrm{m}$.

While the average size can be determined quite accurately by scattering from a suspension, this is not the case for the standard deviation of the size distribution, $\sigma_{P}$. Our best estimate of $\sigma_{P}$ based on light scattering is $0.029 \mu \mathrm{m}$ while, as discussed in section 5 , the value obtained by electron microscopy is about $0.0095 \mu \mathrm{m}$. We suspect that the major cause of the discrepancy is the contribution of scattered light from off-size particles and agglomerated doublets. The off-size particles are excluded from the determination of $\sigma_{P}$ by electron microscopy and the doublets are treated as pairs of individual spheres.

\section{Optical Array Sizing}

Array sizing by optical microscopy provides an independent measure of $D_{\mathrm{n}}$ since the technique is based on geometrical optics rather than diffraction theory. This is one of the oldest techniques for the measurement of average size of micrometer-sized particles. Perrin [25] used this technique in his study of Brownian motion, from which Avogadro's number was first determined. Its ability to produce accurate values for the average diameter results from a combination of a highly accurate length standard for optical microscopy and the fact that monosize polystyrene spheres can form close-packed, two-dimensional hexagonal arrays. The microscopic image of a row of micrometer-size spheres consists of a string of circular images blurred by diffraction as indicated in figure 8; however, the center of the sphere can be pinpointed with considerable accuracy. In fact, when rows of, say, 10 spheres are used, the potential exists for finding the average size of $1 \mu \mathrm{m}$ spheres to a resolution of about $1 \%$ of the sphere diameter. Our methods for preparing the arrays and performing the measurements are similar to those described by Kubitschek [26].

\subsection{Formation of Two-Dimensional Arrays}

To minimize non-volatile residue, the particles were "washed" with 18-megohm-centimeter deionized water filtered with a $0.2 \mu \mathrm{m}$ pore size pleated membrane filter. The washing consisted of pouring off the supernatant after allowing the particles to settle and then adding filtered, deionized water. The particle concentration was about $0.5 \%$ by mass. A drop of the particle suspension was placed at one end of a microscope slide and smeared out by means of a second slide held such that one of its long edges would wipe slowly over the first slide. The evaporating water sweeps the particles inward though it appears that surface tension is responsible for the small scale motion leading to the ordered array. In the early phase of evaporation, the particles tend to congregate in assemblies similar in shape to ice floes in a freezing ice field. As the evaporation continues, the spheres arrange themselves into well-ordered hexagonal arrays, with rows $10-50$ spheres long. This ordering occurrs too rapidly to be observed by eye.

The ordered arrays extending perhaps 7 spheres in one direction and 20 in another are separated by microcracks as seen in figure 9. The cracks occur during the drying when an array field is pulled apart perhaps to relieve a stress on the array. Another type of crack, also illustrated in figure 9, is much shorter and is V-shaped. It seems to indicate intensive drying within an array 


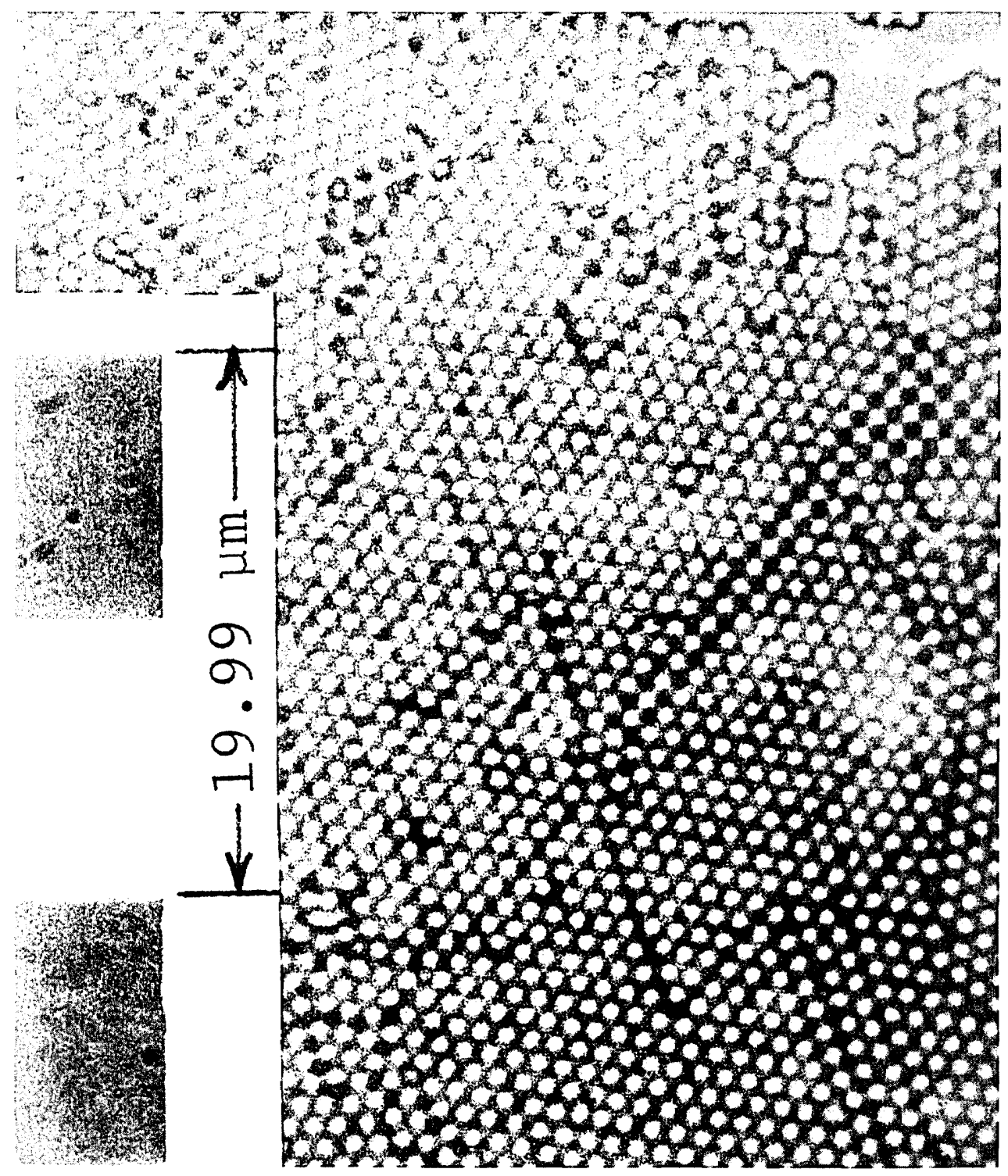

Figure 8-Hexagonal array of nominal one- $\mu \mathrm{m}$ polystyrene spheres (Standard Reference Material 1690).

field. Close inspection shows that such cracks can be detected when they are about $0.05 \mu \mathrm{m}$ wide, and they are easily visible when the width exceeds $0.1 \mu \mathrm{m}$.

\subsection{Measurement of Arrays}

The arrays were measured by scaled microphotography rather than by microscopy with a graduated eyepiece. Microphotography has the advantage of providing a permanent record from which array defects such as microcracks can be accounted for. The image distortion in the optical train was measured to be less than $0.2 \%$, and scale changes from repeated focusing were $0.1-0.2 \%$. The microscope was a Zeiss Universa $1^{1}$ with Polaroid attachment and with a $63 \times, 0.90$ N.A. objective.
The micrographs, with a typical scale of $1.6 \mathrm{~mm}$ per $\mu \mathrm{m}$, were measured to $0.05-0.1 \mathrm{~mm}$. The magnification calibration and the image distortion were measured using a $19.990 \pm 0.005 \mu \mathrm{m}$ linewidth spacing on SRM 474 Line Width Standard from NBS.

The arrays were prepared on six microscope slides. One to three arrays were measured on each slide for a total of 12 arrays. For each array, 15 row lengths were measured. The number-average diameter for a single row is determined by dividing the length of the row, $L$, by the number of spheres in the row minus one, $N-1$.

$$
D_{\mathrm{r}}=\frac{L}{N-1}
$$

The -1 results from using the center-to-center row length rather than the edge-to-edge row length. 

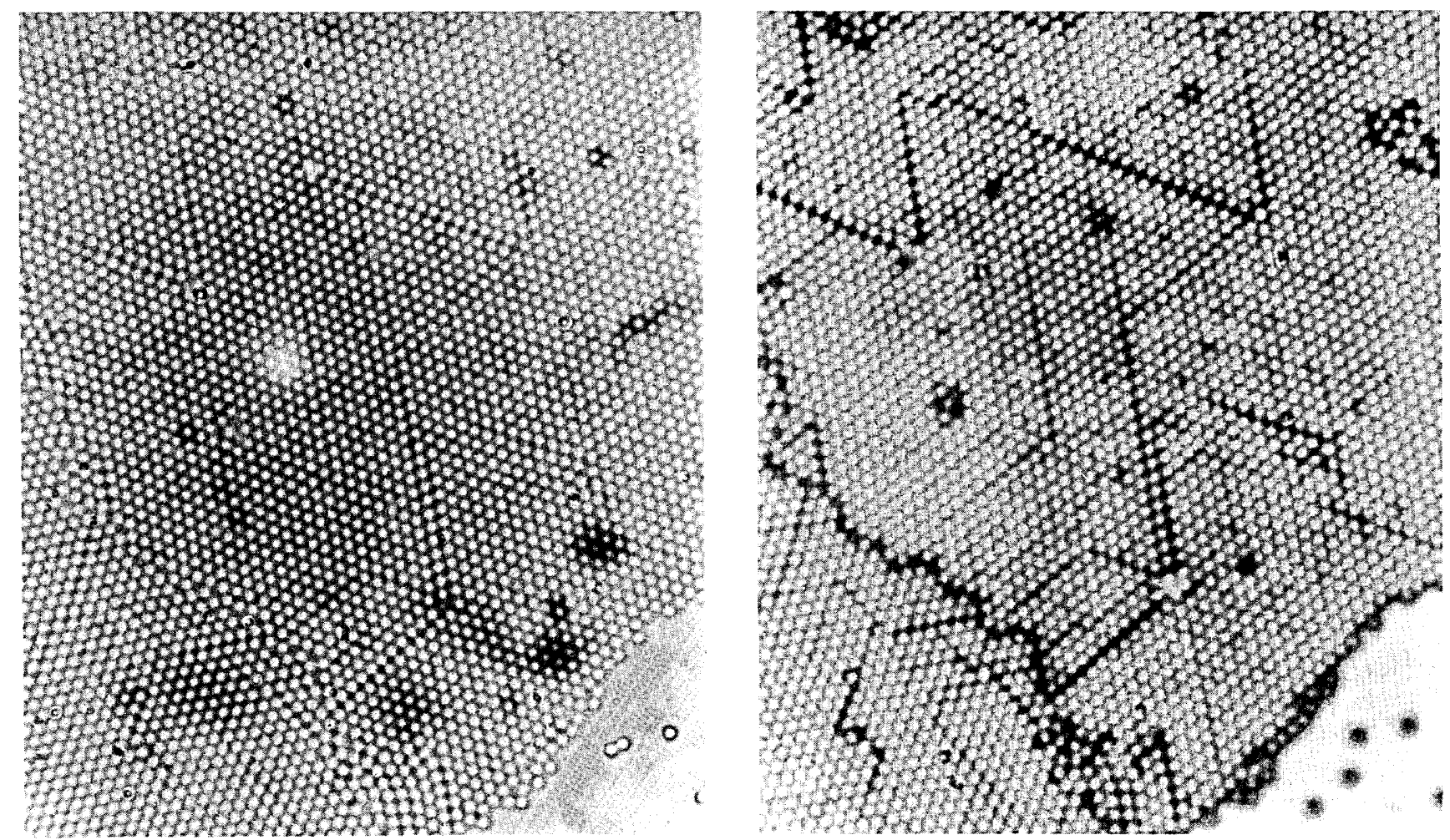

Figure 9-The same field of view is slightly defocussed for the photograph on the right to allow better visualization of the microcracks.

In our measurements the row length varied from 10 to 30 spheres.

\subsection{Results}

The results for two arrays are shown in table 7. Measurements along each of the three lattice directions are included. In some cases the row lengths in different

Table 7. Measured row lengths for two arrays.

\begin{tabular}{cccccccc}
\hline \hline & \multicolumn{3}{c}{$\begin{array}{c}\text { Row Length Readings } \\
(\mathrm{mm})\end{array}$} & \multicolumn{2}{c}{$\begin{array}{c}\text { Row Average Diameter } \\
(\mu \mathrm{m})\end{array}$} & $\begin{array}{c}\text { Orientation } \\
\text { Average } \\
(\mu \mathrm{m})\end{array}$ \\
Slide & & & & & & \\
No. & $0^{\circ}$ & $120^{\circ}$ & $240^{\circ}$ & $0^{\circ}$ & $120^{\circ}$ & $240^{\circ}$ & \\
\hline 3 & 28.6 & 29.0 & 29.15 & & & & \\
& 28.8 & 29.0 & 29.15 & & & & \\
& 28.9 & 28.0 & 29.2 & 0.891 & 0.901 & 0.896 & 0.896 \\
& 28.85 & 28.95 & 29.1 & & & & \\
& 28.9 & 28.9 & 29.1 & & & & \\
6 & & & & & & & \\
& 14.55 & 14.55 & 14.7 & & & & \\
& 14.5 & 14.55 & 14.7 & & & & \\
& 14.5 & 14.5 & 14.7 & 0.898 & 0.898 & 0.908 & 0.901 \\
& 14.5 & 14.4 & 14.7 & & & & \\
& 14.5 & 14.55 & 14.6 & & & & \\
\hline
\end{tabular}

directions differ by as much as $0.2 \mathrm{~mm}$ for an 11-sphere array leading to differences in the average diameter of $0.01 \mu \mathrm{m}$. The reason for this anisotropy is not clear. Kubitschek [26] attributes this effect to undetected microcracks. If the cause were a single microcrack, then it would be on the order of 0.1 to $0.2 \mathrm{~mm}$ and would thus have been detected. For those arrays located at the periphery of the ordered region, we found that in general the average diameters calculated from rows parallel to the edge were somewhat larger than values calculated from rows in the other two directions, suggesting looseness in that direction. The drying speed of the array also seemed to have some influence.

The row-average diameter, $\bar{D}_{\mathrm{r}}$, is obtained as a simple average of the values $D_{\mathrm{r}}(i)$ for the $R$ rows.

$$
\bar{D}_{\mathrm{r}}=\frac{\sum_{i=1}^{R} D_{\mathrm{r}}(i)}{R}
$$

The standard deviation in $D_{\mathrm{r}}, \sigma_{D_{\mathrm{r}}}$ is given by

$$
\sigma_{D_{\mathrm{r}}}=\frac{\sum_{i=1}^{R}\left(D_{\mathrm{r}}(i)-\bar{D}_{\mathrm{r}}\right)^{2}}{R-1}
$$

A summary of the values of $\bar{D}_{\mathrm{r}}$ and $\sigma_{D_{\mathrm{r}}}$ for each of 12 arrays, which includes 240 rows, is given in table 8 . The 
Table 8. Array sizing results for SRM 1690 .

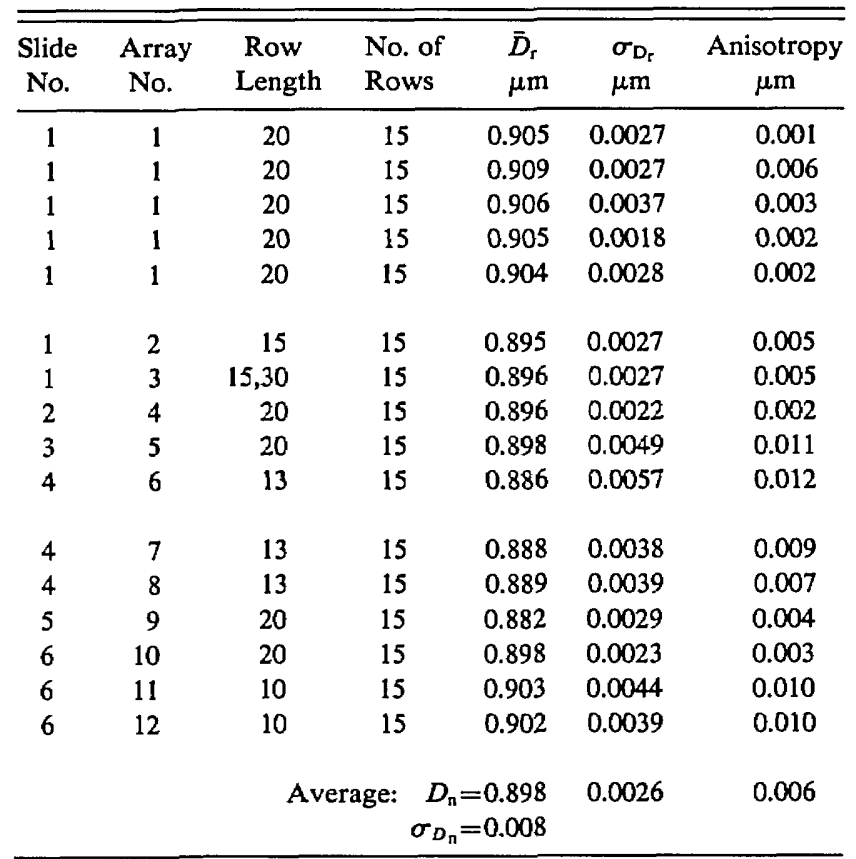

average of all the values of $\bar{D}_{\mathrm{r}}$ in table 8 , defined as $D_{\mathrm{n}}$, is 0.898 with a standard deviation among the row averages, $\sigma_{D_{\mathrm{n}}}$, of $0.008 \mu \mathrm{m}$. The anisotropy, defined as the range in the row-averaged diameters in the three lattice directions of an array, is found to have an average value of $0.006 \mu \mathrm{m}$. This is comparable in magnitude to $\sigma_{D_{\mathrm{n}}}$.

It is also possible to determine the standard deviation of the size distribution, $\sigma_{P}$, from $\sigma_{D_{\mathrm{n}}}$. Assuming the spheres to be randomly distributed along a row in terms of particle size, it can be shown that the $\sigma_{P}$ is related to $\sigma_{D_{r}}$ by the expression,

$$
\sigma_{P}=\sqrt{N-1} \sigma_{D_{r}}
$$

where $N$ is the number of spheres in a row.

In arriving at this result, one makes use of the fact that the variance of the sum of random variables (the row length) is the sum of the variances of the individual random variables (sphere diameter). The resulting value, $0.013 \mu \mathrm{m}$, has a statistical uncertainty of about $\pm 0.003 \mu \mathrm{m}$ and is somewhat larger than the value obtained by electron microscopy, $0.0095 \mu \mathrm{m}$.

\subsection{Error Analysis}

While the experimental technique of optical array sizing is relatively simple compared to light-scattering measurements, there are more potential sources of uncertainty, both random and systematic. The random component of the total uncertainty is related to the standard deviation of the average diameters for the arrays measured, $\sigma_{D_{\mathrm{n}}}$. We estimate the magnitude of the various components of the random error including image distortion, readout of microphotographs, local motion in the photographic material, and anisotropy. These are combined and compared with $\sigma_{D_{\mathrm{n}}}$. There are also a number of systematic errors arising from the finite width of the size distribution, microcracks, solute impurities, and shape distortion.

Image distortion results in the length scale changing over the photographic field of view. Because of axial symmetry of the optical train, it needs to be measured in relation to one plane containing the optical axis. The Standard Reference Material 474 Line Width Standard was used as a two-line resolution target.

A series of 150 measurements was made, covering five photographs, each with 10 areas and each area being measured three times. The result was a scale factor of $0.619 \pm 0.001 \mu \mathrm{m} / \mathrm{mm}$ ( - at center, + at edge). Using one scale value for the entire field of view introduces an image distortion error, $\sigma_{1}$, no greater than $0.002 \mu \mathrm{m}$.

Row lengths were measured to $0.05-0.1 \mathrm{~mm}$ (0.03-0.06 $\mu \mathrm{m})$ using a film scale. The rows contained 10-20 spheres; thus the error contribution to the average diameter value $(1.0 \mu \mathrm{m}$ nominal) was 0.003 to $0.006 \mu \mathrm{m}$. In practice the film readout error, $\sigma_{2}$, was held below $0.005 \mu \mathrm{m}$.

It is known that slight motion can occur in photographic materials primarily as a result of uneven drying. In the Polaroid material used here, there was no detectable motion at the $0.05 \mathrm{~mm}$ level. The error contribution, $\sigma_{3}$, is thus less than $0.003 \mu \mathrm{m}$ for 20 -sphere rows.

The anisotropy in the array will also manifest itself as a component of the random error, $\sigma_{4}$. We estimate this effect as half the range in the average anisotropy, $0.003 \mu \mathrm{m}$.

We obtain a combined estimate of all the sigmas by using the formula

$$
\sigma_{\mathrm{t}}^{2}=\sum_{i=1}^{4} \sigma_{i}^{2}
$$

In using this standard formula, we are assuming the errors to be independent. The resulting value of $\sigma_{t}$, $0.007 \mu \mathrm{m}$, is comparable to $\sigma_{D_{n}}$ so our error budget accounts for the observed value of the random error. The random component of the overall uncertainty based on 16 sets of measurements is found to be $0.0044 \mu \mathrm{m}$ based on the formula

$$
R=t_{15}(0.025) \frac{\sigma_{D_{\mathrm{n}}}}{\sqrt{16}}
$$

It is thought that the mean distance between particle centers in monodisperse arrays, such as those shown in figure 8 , is greater than the mean particle diameter be- 
cause many smaller particles fail to be in contact with all of their neighbors. Thus, gaps in the array add to the length of a line of particles, causing an overestimate of their true mean diameter. For hexagonal arrays formed from machined washers of known size, Kubitschek [27] found that the overestimate $\Delta D$ for particles with standard deviation $\sigma_{P}$ was given by

$$
\Delta D=0.46 \sigma_{P} .
$$

For our case, $\sigma_{P}$ obtained from electron microscopy is $0.0095 \mu \mathrm{m}$, so the resulting systematic error, $\delta_{\mathrm{w}}$, is $-0.0044 \mu \mathrm{m}$. We have chosen to treat this effect as a systematic error rather than correcting for it, because there has been no direct verification of eq (20) for micrometer-size spheres.

A second source of systematic error arises from microcracks. When a row is measured across one or two undetected cracks, one can expect an error contribution to the average diameter of about $0.01 \mu \mathrm{m}$ for rows 10 spheres long, and less for longer rows. We take as our error estimate, $\delta_{\mathrm{C}}$, a value of $-0.005 \mu \mathrm{m}$.

The presence of impurities in the polystyrene suspensions is another source of error. Assuming that the emulsifier and inorganic impurities form a uniform coating around the spheres when the water evaporates, one finds the following expression for the thickness, $t$, of the coating

$$
t=\frac{C_{1} D}{6 C}
$$

where $C$ is the volume concentration of the polystyrene and $C_{1}$ the concentration of the non-volatile impurities. For our case the non-volatile impurity concentration was about two orders of magnitude lower than the volume concentration of the polystyrene spheres, and the maximum resulting error is about $0.003 \mu \mathrm{m}$.

An attempt was made to detect this effect by using one sample of the stock solution and a different one in which the original solute impurity content had been reduced by an order of magnitude by settling, decanting, and addition of filtered, deionized water. The measured diameter for the second sample was slightly less, by $0.004 \pm 0.003 \mu \mathrm{m}$. Therefore, all reported measurements were performed on samples with reduced impurity content. We estimate the error resulting from residual nonvolatile impurity of washed particles, $\delta_{\mathrm{I}}$, as $0.001 \mu \mathrm{m}$.

Two other potential systematic errors include the effect of unequal-size spheres on a flat substrate and the effect of curved rows. If we have a row of spheres of unequal diameters, the centers of the spheres will not be on a straight line and the row length will be less than the sum of the diameters. If the size distribution is not too broad $\left(\sigma_{P} / D<0.03\right)$, the error in the calculated average diameter, $\sigma_{P}{ }^{2} / 2 D^{2}$, is small (less than $0.001 \mu \mathrm{m}$ ) compared to other errors.

Occasionally arrays will exhibit curved or wavy rows. For typical curvatures, replacing the arc by the chord introduces only a small error (on the order of $0.001 \mu \mathrm{m})$ in the measured diameter. However, we found that curved rows are sometimes as much as $1 \%$ longer than the corresponding straight rows found in the same array. This suggests the presence of slack in the curved rows, and therefore such rows were omitted from our measurements.

It is possible that spheres in contact flatten slightly. We made an attempt to measure the average size of isolated spheres and arrays of spheres with a transmission electron microscope, but we were not able to prepare arrays on TEM grids. There were instances of particles in contact as in figure 10 , and from measuring

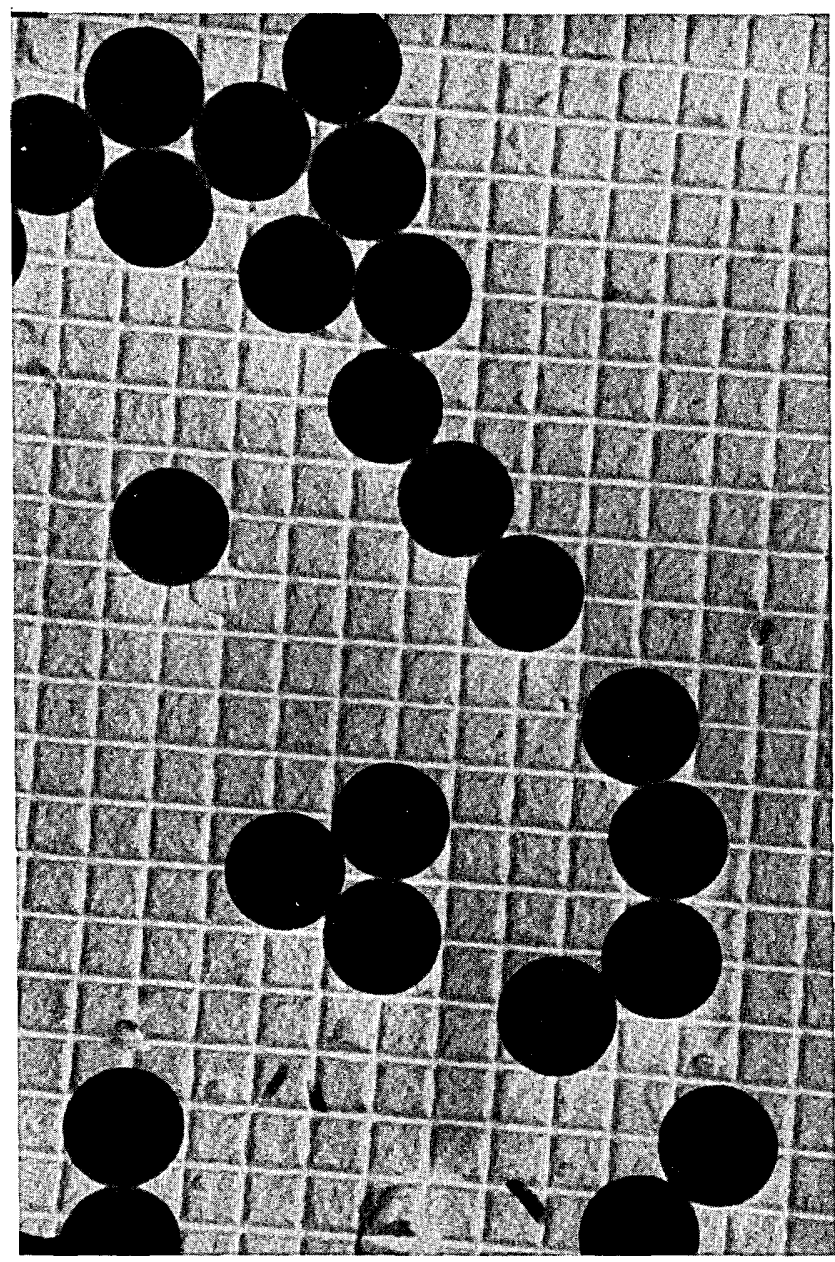

Figure 10-Electron micrograph of nominal one- $\mu \mathrm{m}$ polystyrene spheres (SRM 1690) at a magnification of $10,000 \times$. The spacing for the diffraction grating lines is $0.46 \mu \mathrm{m}$. 
such particles we found that the flattening effect is less than $0.005 \mu \mathrm{m}$. More quantitative measurements are necessary to refine $\delta_{\mathrm{F}}$.

As in the error analyses in the previous sections, we obtain the combined uncertainty from the sum of the random error, $R$, and the absolute values of the systematic errors,

$$
U_{\mathrm{T}}=R+\left|\delta_{\mathrm{W}}\right|+\left|\delta_{\mathrm{C}}\right|+\left|\delta_{\mathrm{I}}\right|+\left|\delta_{\mathrm{F}}\right|
$$

We obtain a value of 0.020 for $U_{\mathrm{T}}$ with the overall average value for $D_{\mathrm{n}}$ of $0.898 \mu \mathrm{m}$.

\section{Measurements of Size Distribution/Concentration}

We have used transmission electron microscopy, flow-through electrical-sensing zone counter measurements, and optical microscopy to obtain more detailed information about the size distribution. Using these techniques, we measure $\sigma_{P}$, the standard deviation of the size distribution, the fraction of off-size particles, and the fraction of agglomerated doublets. Our only direct measure of number concentration was obtained with electrical sensing zone counter measurements.

\subsection{Transmission Electron Microscopy}

The sample was prepared for electron microscopy by evaporating a small drop of diluted suspension of the spheres on a diffraction grating replica mounted on a TEM grid. Three grids were prepared from one SRM 1690 vial. The grids were coated with approximately 20 $\mathrm{nm}$ of carbon and then examined with a JEOL 200CX electron microscope ${ }^{1}$ at an accelerating potential of 100 $\mathrm{KV}$ and at a nominal 10,000 $\times$ magnification. The particle size was measured directly from the negative using a $7 \times$ magnifier with an accurate millimeter scale reticule. A series of concentric circles on the reticule enabled quick location of the sphere diameter. A typical micrograph is shown in figure 10.

As mentioned in the introduction, we had hoped to make an accurate determination of the average particle size from the TEM measurements. However, we found significant variability in the commercially available grating replicas in regard to the line spacing even among those from the same supplier. We also found variations as much as $3 \%$ in the line spacing in different regions of the same grating. So from our assessment, transmission electron microscopy is not competitive with the light scattering and array sizing measurements for accurate particle size measurement without a suitable calibration artifact.
However, the high resolution of the TEM makes it suitable for the measurement of the standard deviation of the narrow size distribution. The size distribution typically consists of a very sharp central peak plus broadly distributed off-size particles. The inclusion of the off-size particles in the data analysis will greatly broaden the apparent value of $\sigma_{P}$. In one case $\sigma_{P}$ changed from 0.031 to 0.011 as a result of removing three off-size particles from a sample of 102 particles. It is, therefore, important to have a systematic procedure for rejecting off-size particles. We used a discordancy test [28] based on the sample kurtosis as the test statistic,

$$
\text { sample kurtosis }=\frac{N_{i=1}^{N}\left(D_{i}-D_{\mathrm{n}}\right)^{4}}{\left(\sum_{i=1}^{N}\left(D_{i}-D_{\mathrm{n}}\right)^{2}\right)^{2}} .
$$

If the sample kurtosis exceeds a value of about 3.77 for a 100-sphere sample, then one or more spheres are offsize at the $5 \%$ level of discordancy. The spheres with diameters farthest from the average size are eliminated consecutively until the sample kurtosis reaches the appropriate value. The particles at the edge of the field of view were eliminated because of evidence of magnification distortion in this region.

Using a common magnification for all the micrographs resulted in a smaller value of $\sigma_{P}$ than the value obtained using a magnification for each photograph derived from the line spacing on the diffraction grating replica. The smaller value of $\sigma_{P}$ is the better value because experimental errors only tend to increase $\sigma_{P}$. The constancy of the magnification was to be expected, since all the micrographs were taken at one magnification setting over a period of a few hours. We obtained values of $0.0083 \mu \mathrm{m}$ and $0.0106 \mu \mathrm{m}$ for $\sigma_{P}$ based on samples of 59 and 99 particles. We take the mean value of 0.0095 as our estimate of $\sigma_{P}$.

The major source of uncertainty in $\sigma_{P}$ is statistical in origin. The sample variance has a $\chi^{2}$ distribution, and for a sample size of 100 there is an uncertainty of about $\pm 13 \%$ at the $95 \%$ confidence level. The size distribution is represented as a histogram in figure 11.

Other information obtained from TEM includes the asphericity of the particles and the fraction of "new" particles. The asphericity, $\Delta_{s}$, is defined as

$$
\Delta_{s}=\left(\frac{D_{\max }-D_{\min }}{\tilde{D}}\right) \times 100 \% .
$$

The diameter measurements were made every $45^{\circ}$ for particles located near the center of the field of view. The asphericity is small, $0.6 \% \pm 0.3 \%$, with large uncertainty because of the resolution limit of the TEM.

We found a total of eight particles with diameters less than $0.5 \mu \mathrm{m}$ out of some 1350 particles $(0.6 \%)$. As ex- 


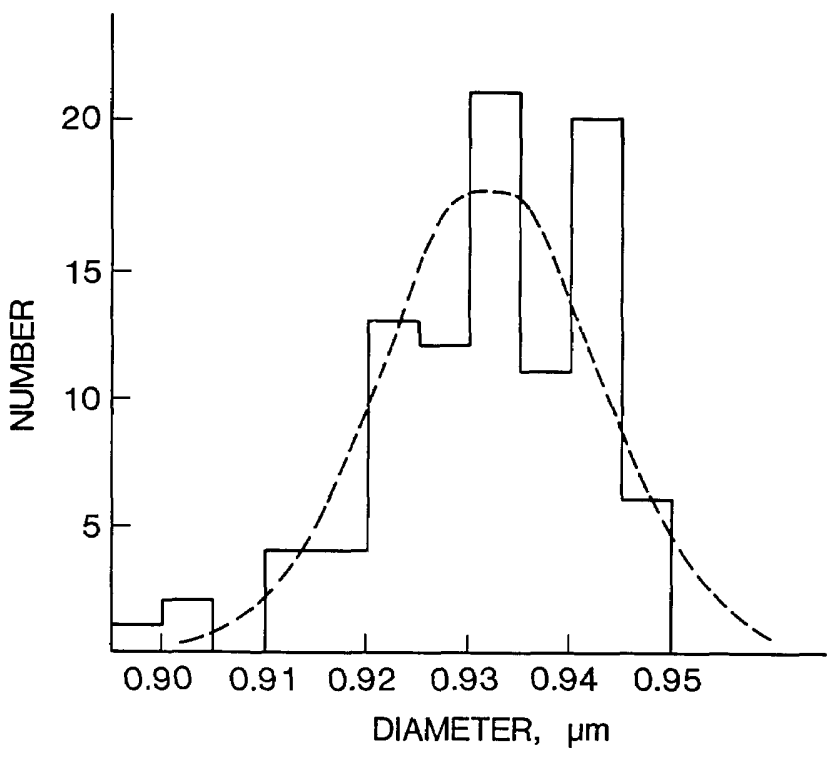

Figure 11-Size distribution of nominal one- $\mu \mathrm{m}$ polystyrene spheres (with off-size particles excluded) obtained by transmission electron microscopy. The dashed curve is the normal distribution with $D_{\mathrm{n}}$ $(0.932 \mu \mathrm{m})$ and $\sigma_{P}(0.0106 \mu \mathrm{m})$ obtained from the experimental data.

plained in the Appendix, it is possible to nucleate a new population of particles in the process of using seed particles to produce larger particles. We believe that this nucleation process is responsible for the small off-size spheres.

\subsection{Electrical Sensing Zone-Counter Measurements}

We used the Coulter ZB Counter with Coulter Channelyzer Pulse Sizer ${ }^{1}$ to measure the fraction of doublets, the fraction of large off-size particles, and the number concentration, and to estimate the variability of $D_{\mathrm{n}}$ in the SRM 1690 samples. To apply this technique, the particles must be prepared in an electrolyte solution at a concentration of about $1 \%$ by mass. The technique consists of measuring the electrical current due to the electrolyte flowing through an orifice; in our case, 30- and $19-\mu \mathrm{m}$ diameter orifices were used. As a particle flows through the orifice, the current is reduced by an amount approximately proportional to the volume of the particle [29].

For our application, we used nominal 0.9- and 2- $\mu \mathrm{m}$ size polystyrene spheres for a particle size calibration of the instrument, and then used the Coulter Counter to measure the concentration and size distribution. The Coulter Counter is not an absolute sizing instrument.

It was found that if the polystyrene spheres were directly diluted with electrolyte, then about $10 \%$ agglomerated doublets resulted from the combined effects of high particle concentration and the reduction in electrostatic repulsion between the particles. The agglomer- ated doublets were minimized by first diluting with filtered, deionized water (dilution factor $2.5 \times 10^{3}$ ) and then diluting by another factor of 10 with the electrolyte solution. The percentage of doublets was found to be $1.5 \pm 0.4 \%$ of the total population for fresh samples, compared to about $1 \%$ obtained by visual microscopy of the suspended particles diluted without electrolyte. Based on the limited sample size, there does not appear to be any significant difference in the fraction of doublets between the sample diluted without electrolyte and the fresh samples diluted with electrolyte at the last step.

By changing the electronic settings of the Coulter Counter, it was possible to estimate the percentage of large single particles with diameters in the range of $2-6$ $\mu \mathrm{m}$. The observed count was on the order of 200 out of a total population of about 235,000 particles, which corresponds to slightly less than $0.1 \%$.

Based on the measured number-concentration of two diluted samples for particles in the 0.6 to $1.4 \mu \mathrm{m}$ size range, we obtain number concentrations of the undiluted samples of 1.18 and $1.45 \times 10^{10}$ particles $/ \mathrm{cm}^{3}$. These values agree well with a calculated value of $1.25 \times 10^{10}$ particles $/ \mathrm{cm}^{3}$ based on a concentration of $0.5 \%$ by mass of the polystyrene spheres, a particle density of $1.052 \mathrm{~g} / \mathrm{cm}^{3}$, and a particle size of $0.9 \mu \mathrm{m}$.

Diluted samples were prepared from each of 10 SRM vials and two portions of each sample were sized with the Coulter Counter using the $30-\mu \mathrm{m}$-diameter orifice. A typical size distribution based on sizing about 85,000 particles is given in figure 12. A complete listing of the

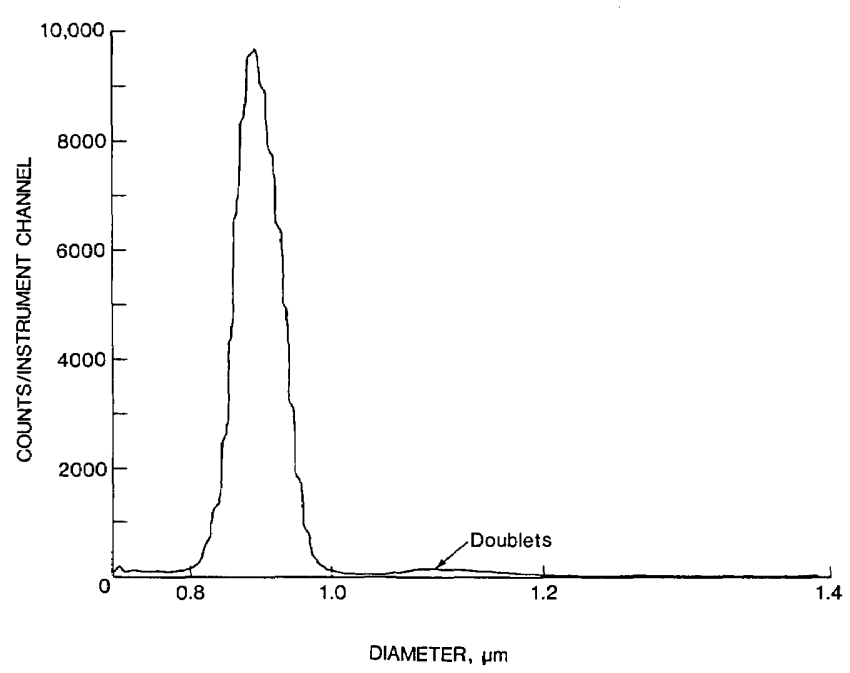

Figure 12-Size distribution of SRM 1690 obtained by a Coulter Counter for aperture current, amplification, and lower threshold settings of $1 / 3,1 / 2$, and 100 respectively. The instrument output is linear with respect to particle volume and consequently appears stretched when plotted versus particle diameter. 
average particle size, $D_{\mathrm{n}}$, and the standard deviation of the central peak are given for each sample in table 9. The standard deviation associated with 10 independent determinations of $D_{\mathrm{n}}$ has a value of $0.0027 \mu \mathrm{m}$. This value is approximately three times greater than the value obtained by light scattering and is due to instrumental noise rather than sample-to-sample variability. Even when measurements are made on the same sample such as $\mathrm{F} 1$ to $\mathrm{F} 4$, variations in $D_{\mathrm{n}}$ as large as $0.005 \mu \mathrm{m}$ are observed.

Table 9. $D_{\mathrm{n}}$ and $\sigma_{P}$ for Coulter Counter measurements of SRM 1690.

\begin{tabular}{lrrrr}
\hline & \multicolumn{2}{c}{ Portion 1 } & \multicolumn{2}{c}{ Portion 2} \\
Sample & $D_{\mathrm{n}, \mu \mathrm{m}}$ & $\sigma_{P}, \mu \mathrm{m}$ & $D_{\mathrm{n}}, \mu \mathrm{m}$ & $\sigma_{P}, \mu \mathrm{m}$ \\
A & 0.9226 & 0.0370 & 0.9239 & 0.0336 \\
B & 0.9225 & 0.0380 & 0.9240 & 0.0344 \\
C & 0.9266 & 0.0351 & 0.9286 & 0.0349 \\
D & 0.9226 & 0.0336 & 0.9240 & 0.0339 \\
E & 0.9264 & 0.0351 & 0.9251 & 0.0357 \\
FI $1^{\mathrm{a}}$ & 0.9308 & 0.0392 & 0.9302 & 0.0332 \\
F2 & & & 0.9251 & 0.0329 \\
F3 & & & 0.9246 & 0.0326 \\
F4 & & & 0.9245 & 0.0325 \\
G & 0.9245 & 0.0328 & 0.9247 & 0.0356 \\
H & 0.9231 & 0.0333 & 0.9222 & 0.0338 \\
I & 0.9223 & 0.0338 & 0.9236 & 0.0340 \\
J & 0.9226 & 0.0323 & 0.9228 & 0.0311 \\
\hline
\end{tabular}

${ }^{a}$ The numerals refer to repeat measurements of the same sample.

The average value of the standard deviation of the size distribution, $0.033 \mu \mathrm{m}$, for the central peak based on Coulter Counter measurements is more than a factor of three greater than the value obtained by electron microscopy. The primary cause of this broadening is the variation in the electrical pulse resulting from different particle trajectories through the orifice. The amplitudetime history of the electrical pulse for a particle moving near the aperture wall is different from that of a particle moving near the centerline. Thom [30] demonstrated this effect on a model system with plastic washers and Spielman and Goren [31] demonstrated that the resolution of the Coulter Counter could be improved by hydrodynamically focusing the particles through the center of the orifice. The Coulter Counter we used did not have hydrodynamic focusing, but it did have a pulse analyzer for rejecting counts in part due to particles with atypical trajectories.

\section{Summary}

The values of $D_{\mathrm{n}}$ and the associated uncertainty for three techniques are: $0.895 \pm 0.007 \mu \mathrm{m}$ for light scattering from particles suspended in water, $0.900 \pm 0.012 \mu \mathrm{m}$ for single-particle light-scattering measurements, and $0.898 \pm 0.020 \mu \mathrm{m}$ for optical array sizing. For each tech- nique there is a consistency test in addition to a quantitative error analysis. For the single-particle lightscattering measurements, the particle diameter obtained for the incident light polarized in the vertical direction $(0.903 \mu \mathrm{m})$ agreed well with the diameter obtained for light polarized in the horizontal direction $(0.898 \mu \mathrm{m})$. For light scattering from a particle suspension, the particle diameter obtained with two different laser sources, $\mathrm{He}-\mathrm{Ne}(0.633 \mu \mathrm{m})$ and He-Cd $(0.442 \mu \mathrm{m})$ differed by only $0.001 \mu \mathrm{m}$. In the case of array sizing, six independent samples were prepared and the variation in the average particle diameter, 0.882 to $0.903 \mu \mathrm{m}$, was less than the overall uncertainty.

We use $0.895 \mu \mathrm{m}$ as the certified value because of the lower uncertainty associated with light scattering from a suspension. Another advantage of this value is that the measurements characterize the particles in the same form as provided in the SRM 1690, this is, in suspension rather than as a dried sample where there is the possibility of a coating of non-volatile impurities on the surface. It is reassuring that the variability among the three values of $D_{\mathrm{n}}$ is less than the uncertainty associated with any one technique. We expect that our estimates of uncertainty are, if anything, conservative. The value obtained by Dow Chemical Co. for $D_{\mathrm{n}}$ by TEM, $0.914 \mu \mathrm{m}$, is about $2 \%$ greater than our certified value.

In table 10 we summarize all the information regarding the size distribution obtained by our measurements. Only for $D_{\mathrm{n}}$ and the index of refraction have detailed error analyses been made. The different features of the global size distribution extending from 0.1 to $6 \mu \mathrm{m}$ are indicated qualitatively in figure 13 .

A wide range of values is found for the standard deviation of the size distribution, $\sigma_{P}$, extending from

Table 10. Summary of results for SRM 1690.

\begin{tabular}{ll}
\hline \hline$D_{\mathrm{n}}, \mu \mathrm{m}$ & $0.895 \pm 0.007^{\mathrm{a}}$ light scat. from suspension (LSS) \\
& $0.900 \pm 0.012$ single part. scattering (SPS) \\
& $0.898 \pm 0.020$ opt. array sizing (OAS) \\
& \\
$\sigma_{P}, \mu \mathrm{m}$ & $\begin{array}{l}0.0095 \pm 0.002 \text { TEM, } 0.010 \text { SPS, } 0.013 \text { OAS, } 0.029 \mathrm{LSS}, \\
0.033 \text { Coulter Counter }\end{array}$
\end{tabular}

\begin{tabular}{|c|c|c|}
\hline$\%$ doublets & $1.5 \pm 0.4$ & Coulter Counter \\
\hline $\begin{array}{l}\% \text { large off-size } \\
\text { particles }\end{array}$ & 0.1 & Coulter Counter \\
\hline$\%$ "new" particles & $0.6 \pm 0.2$ & TEM \\
\hline$\%$ asphericity & $0.6 \pm 0.3$ & TEM \\
\hline number concentration & $1.3 \pm 0.2 \times 10^{10} \mathrm{part} / \mathrm{cm}^{3}$ & Coulter \\
\hline index of refraction & $1.612 \pm 0.009$ & $\begin{array}{l}\text { single particle } \\
\text { scattering }\end{array}$ \\
\hline \multirow[t]{2}{*}{ sample variability } & $0.0005 \mu \mathrm{m}$ & $\begin{array}{l}\text { light scat. from } \\
\text { suspension }\end{array}$ \\
\hline & $0.0009 \mu \mathrm{m}$ & Coulter Counter \\
\hline
\end{tabular}

${ }^{2}$ The first value in each case is the best estimate. 
Figure 13-Global size distribution.

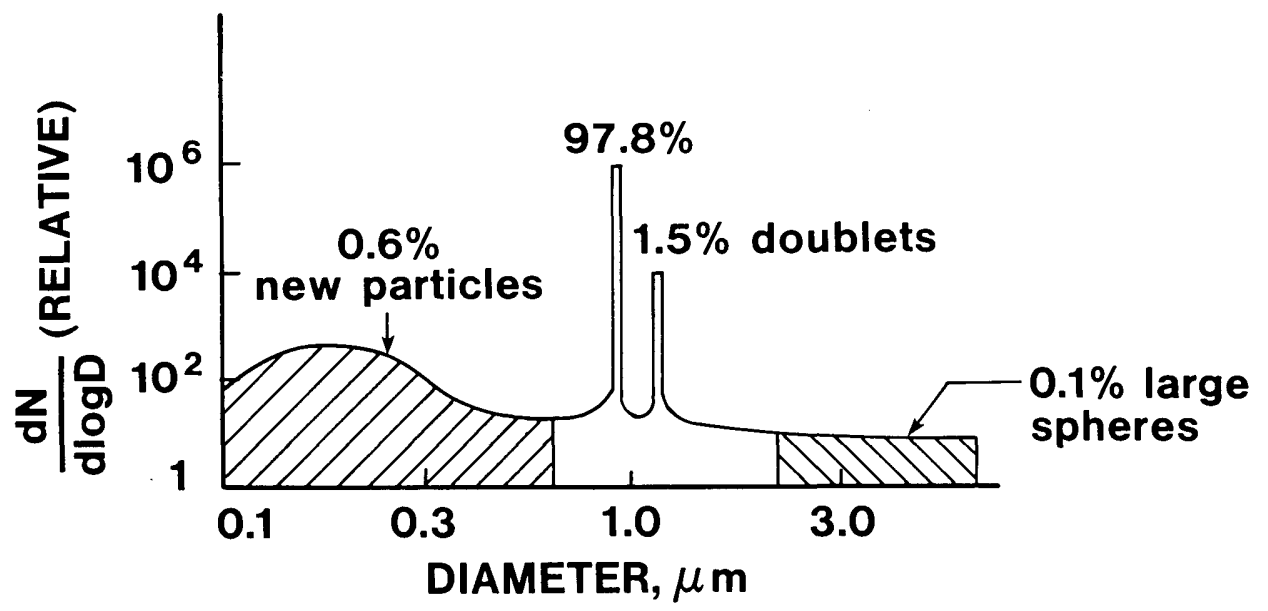

$0.0095 \mu \mathrm{m}$ to $0.033 \mu \mathrm{m}$. This is because each instrument has a sigma associated with repeat measurements of the same objects, $\sigma_{\mathrm{r}}$, and measured values of $\sigma_{P}$ given in table 10 represent a combined effect of the $\sigma$ of the true size distribution and $\sigma_{\mathrm{r}}$. The single-particle scattering measurement has the smallest $\sigma_{\mathrm{r}}, 0.002 \mu \mathrm{m}$, but the statistical uncertainty due to the small number of particles measured (eight) limits the accuracy of $\sigma_{P}$. The best estimate of $\sigma$ comes from TEM with a slightly larger $\sigma_{r}$, about $0.003-0.004 \mu \mathrm{m}$, compared to the single particle measurements, but this is offset by the improved statistics from a much larger sample size (100). The large values of $\sigma_{\mathrm{r}}$ for the other instruments result from the dependence of the electrical pulse on the particle trajectory through the orifice for the Coulter Counter, the presence of doublets and off-size particles for lightscattering measurements of a particle suspension, and array nonuniformities for the case of optical array sizing.

Each measurement method requires a slightly different sample preparation. A necessity in almost all cases is particle free water for dilution. We found $18 \mathrm{megohm}$ $\mathrm{cm}$ deionized water passed through a $0.2 \mu \mathrm{m}$ pore size filter to be adequate for our measurements. Clean glassware is a necessity for diluting the samples and a clean optical cell free of water marks is required to obtain good light scattering data. As described in section 3, we used both detergent and hot, concentrated nitric acid for cleaning our glassware. In the case of diluting a sample, especially if an electrolyte is used, there is always the possibility of promoting agglomeration through the reduction in the electrostatic repulsion between the particles. We found that even for a $1 \%$ by weight solution of sodium chloride little agglomeration occurred in an hour for a particle concentration of about $10^{6} \mathrm{part} / \mathrm{cm}^{3}$ if the electrolyte were introduced only at the final dilution stage as described in subsection 5.2.

\section{Concluding Remarks}

Our measurements have resulted in a particle size standard with an uncertainty in particle diameter of about $0.8 \%$. Modest improvements in sizing accuracy, say a twofold reduction in uncertainty, may be possible with the existing techniques. About half the total uncertainty in the scattering measurements for suspensions is associated with the uncertainty in the refractive index. The refractive index of liquids is known to an uncertainty of a few parts in $10^{5}$, while the uncertainty in the refractive index of the polystyrene spheres is a few parts in $10^{3}$. It is possible that at least a tenfold refuction in the uncertainty in the refractive index of the spheres could be achieved by index matching the spheres with a liquid. A second improvement would be to remove the agglomerated doublets by sedimentation before performing the scattering measurements.

An accurate value of the refractive index of the polystyrene spheres would also improve the sizing capabilities of single particle scattering messurements since there would be only one unknown prameter, the particle diameter, to be determined from the scattering measurements. We have found that tle sizing resolution improves by a factor of two to three if the refractive index is fixed when performing the data analysis. The single greatest source of uncertaity arises from the small number of particles sized (ei,ht); this component of the error could be cut in half if 4 particles were sized.

Half of the uncertainty for optial array sizing results from smaller particles not toucling neighboring particles and from slight flattening of the spheres on contact. Hartman [32] has demonstnted that it is possible to make square arrays of partiles, where, unlike inhexagonal arrays, every partile is in contact with its neighbors. A systematic analsis of such arrays is possible but would be time-consiming because of the rela- 
tively rare occurrence of square arrays. A quantitative analysis of flattening could be made by transmission electron microscopy if arrays could be prepared on TEM grids.

Major improvements in sizing accuracy, say a tenfold reduction in uncertainty, will require a more detailed characterization of the particle structure. Specific structural information of interest includes particle shape, especially in regard to deviation from sphericity, particle inhomogeneity in regard to a variable index of refraction within the particle, and the surface structure both for particles in suspension where an electrical double layer exists and in aerosol form where nonvolatile impurities coat the surface. The pertinent structural information must also be included in the light scattering theory for determining particle size from scattering intensity as a function of angle. Of course, if particles could be made with a high degree of sphericity and low inhomogeneity within the particle, the analysis would be simplified.

Ultimately it is planned to develop particle size standards over the diameter range of 0.1 to $100 \mu \mathrm{m}$. For such a wide range, a variety of techniques will be used. One promising instrument for larger particle sizes involves optical interferometry coupled to an electron microscope. One of us (G.G. Hembree) has developed such an instrument with a piezoelectric stage, the displacement of which is monitored interferometrically with an uncertainty on the order of $0.02 \mu \mathrm{m}$. Another technique for larger particles consists of the measurement of resonances in the polarization ratio of scattered light. Lettieri et al. [33] measured the diameter of droplets in the size range 6 to $12 \mu \mathrm{m}$ to a resolution of $0.003 \mu \mathrm{m}$ using this technique.

Work is currently in progress to develop a nominal $0.3 \mu \mathrm{m}$ particle size standard. In this case, quasielastic scattering is being used in conjunction with transmission electron microscopy with the magnification calibration based on the $0.815 \mu \mathrm{m}$ spheres (SRM 1690).

\section{References}

[1] Swyt, D. A. A lod at techniques for the dimensional calibration of standard meroscopic particles. Natl. Bur. Stand. (U.S.) Spec. Publ. 26)-85 (1983).

[2] Phillips, D. T.; P. J.Wyatt and R. M. Berkman. Measurement of the Lorenz-Miescattering of a single particle: polystyrene latex. J. Colloid'nterface Sci. 34: 159 (1970).

[3] Rowell, R. L.; R. S. larinato, J. W. Parsons, J. R. Ford, K. H. Langley, J. R. Stçe, T. R. Marshall, C. S. Parmenter, M. Seaver and E. B. Iradford. Polystyrene latex particle size by electron microsspy and light scattering. J. Colloid Interface Sci. 69(3): 59-595 (1979).

[4] Bierhuizen, H. W. J., ancG. A. Ferron. Determination of the diameter of two moodisperse lattices by the free fall method and the length of particle arrays. J. Aerosol Sci. 6: 19-21 (1975).

[5] Gulari, Es.; Er. Gulari, Y. Tsunashima, and B. Chu. Photon correlation spectroscopy of particle distributions. J. Chem. Phys. 70: 3965-3972 (1979).

[6] Bonse, J., and M. Hart. Small angle x-ray scattering by spherical particles of polystyrene and polyvinyltoluene. J. Phys. 189: 151 (1966).

[7] Davidson, J. A.; C. W. Macosko and E. A. Collins. Latex particle size analysis I. flow microscopy. J. Colloid Interface Sci. 25: 381-388 (1967).

[8] van den Hul, H. J., and J. W. Vanderhoff. "Clean" monodisperse latexes as model colloids, chapter 1 in Polymer Colloids. R. M. Fitch, ed. New York, NY: Plenum Press (1971) pp. 1-27.

[9] Wyatt, P. J., and D. T. Phillips. A new instrument for the study of individual aerosol particles. J. Colloid Interface Sci. 39(1): 125-135 (1972).

[10] Cooke, D. D., and M. Kerker. Particle size distribution of colloidal suspensions by light scattering based upon single particle counts-polystyrene latex. J. Colloid Interface Sci. 42(1): 150-155 (1973).

[11] McRae, D. D. The refractive index of individual cigarette smoke droplets. J. Colloid Interface Sci. 87: 117-123 (1982).

[12] Davis, E. J., and P. Ravindran. Single particle light scattering measurements using the electrodynamic balance. Aerosol Sci. and Tech. 1: 337-350 (1982).

[13] Bottiger, J. R.; E. S. Fry and R. C. Thompson. Phase matrix measurements for electromagnetic scattering by sphere aggregates in light scattering by irregularly shaped particles. D. W. Shuerman, ed: New York: Plenum Press (1980) pp. 280-290.

[14] Marx, E., and G. W. Mulholland. Size and refractive index determination of single polystyrene spheres. J. Res. Natl. Bur. Stand. (U.S.) 88: 321-338 (1983).

[15] Mie, G. A contribution to the optics of turbid media, especially colloidal metallic suspensions. Ann. Physik 25: 337-445 (1908).

[16] Born, M., and E. Wolf. Principles of optics. Oxford: Pergamon Press (1980).

[17] Kerker, M. The scattering of light and other electromagnetic radiation. New York: Academic Press (1969).

[18] Wims, A. M., and M. E. Myers, Jr. An automated, laser lightscattering photometer $\left(0.1\right.$ to $170^{\circ}$ range) with a single photon counting system. J. Colloid Interface Sci. 39(3): 447-461 (1972).

[19] Matheson, L. A., and J. L. Saunderson. Optical and electrical properties of polystyrene, chapter 11 in Styrene: its polymers, copolymers, and derivatives, R. H. Boundy and R. F. Boyer, ed. New York, NY: Reinhold Publishing Corp. (1952) 524.

[20] Tilton, T. W., and J. K. Taylor. Refractive index and dispersion of distilled water for visible radiation of temperatures 0 to $60^{\circ}$ C. J. Res. Natl. Bur. Stand. (U.S.) 20: 419 (1938).

[21] Starkie, D. Brit. Plastics 19: 46 (1946).

[22] Smart, C. and E. Willis. Determination of refractive indices of polystyrene latices by light scattering. J. Colloid Interface Sci. 25: 577-583 (1967).

[23] Heller, W. and T. L. Pugh. Experimental investigations on the effect of light scattering upon the refractive index of colloidal paticles. J. Colloid Interface Sci. 12: 299-307 (1957).

[24] Napper, D. H., and R. H. Ottewill. Multiple scattering effects in polystyrene latex dispersions. J. Colloid Interface Sci. 19: 72-80 (1964).

[25] Perrin, M. J. Brownian movement and molecular reality, An- 
nales de Chimie et de Physique, $8^{\text {me }}$ series, (1909) translated by F. Soddy. London: Taylor and Francis (1910).

[26] Kubitschek, H. E. Array method of sizing monodisperse particles, in Ultrafine particles, W. E. Kuhn, ed. Wiley (1961) p. 438.

[27] Kubitschek, H. E. Optical calibration of some monodisperse polystyrene latexes in arrays. Nature 192: 1148-1150 (1961).

[28] Barnett, V., and T. Lewis. Outliers in statistical data. New York Wiley (1978) p. 101.

[29] Allen, T. Particle Size measurements. London: Chapman and Hall (1975).

[30] Thom, von R. Vergleichende untersuchungen zur elecktronischen zillvolumen-analyse, AEG-Telefunken report, Ulm, Germany, (around 1970).
[31] Spielman, L. and S. L. Goren. Improving resolution in Coulter counting by hydrodynamic focusing. J. Colloid Interface Sci. 26: 175-182 (1968).

[32] Hartman, A. W. Experimental confirmation of the Kubitschek effect, Journal of Powder Technology, in press.

[33] Lettieri, T. R.; W. D. Jenkins and D. A. Swyt. Sizing of individual optically levitated evaporating droplets by measurement of resonances in the polarization ratio. Appl. Opt. 20: 2799-2805 (1981).

[34] Blackley, D. C. Emulsion polymerisation theory and practice. New York: Wiley (1975).

[35] Verwey, E. J. W., and J. Th. G. Overbeek. Theory of stability of lyophobic colloids. New York: Elsevier (1948).

\section{APPENDIX \\ Preparation and Stability of Polystyrene Sphere Suspensions}

Preparation-The procedure used by Dow Chemical Co. to produce monosize polystyrene spheres is known as emulsion polymerization. A brief description of the general process is provided here, though the specific emulsifying agent and chemical initiator used by Dow are confidential. A more detailed description of emulsion polymerization is given by Blackley [34].

Emulsion polymerization involves four components: water, styrene monomer, an emulsifying agent, and a free radical initiator. A typical emulsifying agent is sodium lauryl sulfate. The emulsifier will permit the dispersion of the stirred monomer phase into a stable system of micrometer-size droplets. At a sufficiently high concentration of emulsifier, hydrated aggregates called micelles, made up of 50 to 100 molecules of emulsifier, will form. The concentration of micelles is approximately six orders of magnitude greater than that of the monomer droplets.

The polymerization reaction begins when the free radical initiator is heated to sufficiently high temperature to dissociate into radical ions. This is around 50 ${ }^{\circ} \mathrm{C}$ for persulfate anions. The sulfate radical anions attack the styrene dissolved in the aqueous phase to form new ion radicals. The polymer chain begins growing by the addition of more styrene molecules to the radicals in the aqueous phase. The polymer-containing radicals soon enter the micelles where propagation of the chain is continued by attack on the monomer within the micelle. The growth of the polymer chain is supported by the rapid diffusion of the styrene from the droplets into the growing micelles. This growth is terminated by reaction with a second free radical. At this point the chain polymer may have reached a molecular weight on the order of $10^{6}$. Later the chain growth is reinitiated by another radical, and this process will continue until most of the styrene is consumed.

While the polymer growth is a chain process, the intermolecular forces and surface tension lead to a spherical shape analogous to a ball of string. The narrowness of the size distribution results from the uniformity of styrene concentration throughout the reactor and the growth time being long compared to the initiation time. The concentration of the initiator and emulsifier are also important for obtaining a narrow size distribution. Too much emulsifier can lead to new particle formation. This is especially true when small polystyrene spheres are used as seed particles for growing larger ones. The growth of $1-\mu \mathrm{m}$-diameter spheres for SRM 1690 required the use of seed particles, and there was evidence of a new generation of small particles.

Stability-The electrostatic charge on the surface of the particles, created by the outward pointing ionic endgroups, is crucial to the stability of the suspension. Changes in the surface layer can lead to agglomeration of the spheres. Dilution and the addition of electrolyte, required for some of the measurement techniques, can affect the stability of the suspension. The VerweyOverbeek theory [35] describes the stability of colloidal suspensions in terms of the electrostatic forces of repulsion between colloidal particles and London-van der Waals forces of attraction. The electrostatic repulsion arises from the surface charge; for example, from the adsorbed emulsifier ions. The repulsion is greatest at the point of contact between the electrical double layers on the two particles. This is on the order of a nanometer from the polymeric surface, since the ionic portion of 
the emulsifier is directed away from the sphere. At greater distances between the particles' centers, the repulsion drops off exponentially. The magnitude of the repulsion depends on the surface particle charge and electrolyte concentration. The London-van der Waals forces are greatest close to the particle surface (a few tenths of a nanometer from the polymeric surface) and are not strongly affected by the surface charge of electrolyte concentration. Of course, there are very strong repulsive forces as the polymeric molecules of two spheres come in contact.

The sum of these three forces leads to a potential with a minimum at short distances, a repulsive barrier due to electrostatic repulsion at greater distances, and possibly a second minimum at relatively large distances, resulting from the longer range of the London-van der Waals forces. If the particle has sufficient energy to penetrate the electrostatic barrier of another particle, the two particles will stay together in an aggregate. An increase in the electrolyte concentration or a decrease in the surface charge density caused by diluting the suspension with electrolyte will lower the repulsive barrier and may lead to agglomeration. Such an effect was a concern in the electrical-sensing zone counter measurments as discussed in subsection 5.2, since the particle suspension was diluted with electrolyte. 